\title{
Different stress responsive strategies to drought and heat in two durum wheat cultivars with contrasting water use efficiency
}

\author{
Alessio Aprile ${ }^{1 *}$, Lenka Havlickova ${ }^{2,3}$, Riccardo Panna ${ }^{1}$, Caterina Marè2 ${ }^{2}$ Grazia M Borrelli ${ }^{4}$, Daniela Marone ${ }^{4}$ \\ Carla Perrotta', Patrizia Rampino ${ }^{1}$, Luigi De Bellis ${ }^{1}$, Vladislav Curn ${ }^{3}$, Anna M Mastrangelo ${ }^{4}$, Fulvia Rizza ${ }^{2}$ \\ and Luigi Cattivelli ${ }^{2}$
}

\begin{abstract}
Background: Durum wheat often faces water scarcity and high temperatures, two events that usually occur simultaneously in the fields. Here we report on the stress responsive strategy of two durum wheat cultivars, characterized by different water use efficiency, subjected to drought, heat and a combination of both stresses.

Results: The cv Ofanto (lower water use efficiency) activated a large set of well-known drought-related genes after drought treatment, while Cappelli (higher water use efficiency) showed the constitutive expression of several genes induced by drought in Ofanto and a modulation of a limited number of genes in response to stress. At molecular level the two cvs differed for the activation of molecular messengers, genes involved in the regulation of chromatin condensation, nuclear speckles and stomatal closure. Noteworthy, the heat response in Cappelli involved also the up-regulation of genes belonging to fatty acid $\beta$-oxidation pathway, glyoxylate cycle and senescence, suggesting an early activation of senescence in this $\mathrm{cv}$. A gene of unknown function having the greatest expression difference between the two cultivars was selected and used for expression QTL analysis, the corresponding QTL was mapped on chromosome 6B.

Conclusion: Ofanto and Cappelli are characterized by two opposite stress-responsive strategies. In Ofanto the combination of drought and heat stress led to an increased number of modulated genes, exceeding the simple cumulative effects of the two single stresses, whereas in Cappelli the same treatment triggered a number of differentially expressed genes lower than those altered in response to heat stress alone. This work provides clear evidences that the genetic system based on Cappelli and Ofanto represents an ideal tool for the genetic dissection of the molecular response to drought and other abiotic stresses.
\end{abstract}

\section{Background}

Heat and drought stress and their combination are the most important stresses experienced by plants and they are responsible of a large fraction of productivity losses [1]. Plants respond to stress with a wide range of modifications leading to changes at morphological, cellular, physiological, biochemical, and molecular level [2,3]. A relevant component of the plant adaptation to stress conditions is dependent on transcriptional changes and the expression of key genes results in enhanced stress tolerance

\footnotetext{
* Correspondence: alessio.aprile@unisalento.it

${ }^{1}$ Department of Biological and Environmental Sciences and Technologies,

University of Salento, Prov.le Lecce Monteroni, I-73100 Lecce, Italy

Full list of author information is available at the end of the article
}

$[4,5]$. Overall, the molecular response of plants to abiotic stress is mediated by a number of molecules involved in signal transduction leading to the activation of specific gene networks resulting from the re-programming of cell expression machinery. To these networks belong genes coding for a variety of proteins involved in DNA remodeling, transcription regulation, protein modifications, etc. [6]. A number of publications described the transcriptional changes induced in response to drought [7-9] and heat $[10,11]$ stresses, however much less is known when plants are simultaneously subjected to drought and heat stress, an event very common under field conditions. Several works indicate that the molecular response to the combination of heat and drought activates networks that are

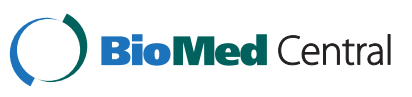


different from those activated by heat or drought stress taken singularly [12-15]. Furthermore, most of the publications cited above have been carried out on seedlings and therefore might not reflect exactly the molecular response of crops exposed to stress when plants are in more advanced growing stages.

Durum wheat is an important cereal crop grown mainly in semi-arid environments (e.g. Mediterranean regions) characterized by water scarcity and high temperatures often occurring at the same time. The two durum wheat cultivars Cappelli and Ofanto, contrasting for many agronomic and physiological traits, have been extensively characterized [16-18]. Measures based on stomata conductance and on grain carbon isotope discrimination from field trials and growth chamber experiments consistently showed a higher water use efficiency (WUE) in Cappelli compared to Ofanto, a finding correlated with a different stomata conductance (lower in Cappelli) over a range of relative soil water contents [17]. A RIL segregating population with a corresponding molecular marker map has also been developed from the cross between Ofanto and Cappelli $[19,20]$ and used to localize QTLs for leaf porosity and chlorophyll content in field conditions [18].

This work reports on a microarray-based transcriptomic analysis carried out on the durum wheat cultivars Cappelli and Ofanto grown to booting stage and subjected to heat, drought and to a combination of drought and heat stresses, conditions similar to the experience of a crop grown in Mediterranean environments and exposed to a terminal heat/drought stress. Furthermore, several selected drought-related genes have been tested in the same cultivars exposed to drought at tillering stage to confirm the constitutive nature of the different stress response strategy detected at booting stage. A gene selected among those characterized by different stress response between the two cultivars was used for an expression QTL analysis and the corresponding QTL was mapped on chromosome $6 \mathrm{~B}$.

\section{Results and discussion}

\section{Sample preparation and hybridization quality}

Plants of two Triticum turgidum subsp. durum cultivars, Ofanto and Cappelli, were grown in controlled conditions and exposed to drought stress (DS), heat stress (HS) and to a combination of heat and drought stresses (CS) when they reached the booting stage. Control (Ctrl) plants were watered to maintain a Soil Water Content (SWC) equal to $28 \%$. DS was imposed by withholding water and allowing the pots to reach a SWC equal to $12.5 \%$, while HS was imposed progressively exposing the plants for two days at $30^{\circ} \mathrm{C}$ followed by additional two days at $34^{\circ} \mathrm{C}$, and subsequently to $40^{\circ} \mathrm{C}$. For CS the two treatments were combined in order to achieve simultaneously
$12.5 \%$ SWC and $40^{\circ} \mathrm{C}$. More details are reported in the Methods section. Table 1 reports the flag leaf temperature and the Relative Water Content (RWC\%) determined on the same plants used for RNA isolation immediately before sampling. Cappelli was characterized by higher leaf temperature compared to Ofanto, a finding in agreement with a lower transpiration capacity due to a lower stomatal conductance as reported by Rizza et al. [17]. Nevertheless, Cappelli showed also a lower RWC compared to Ofanto, a condition that could be explained by a different capacity of water uptake from soil.

Total RNA was isolated from the flag leaves and used for microarray hybridization.

The comparison of the "RNA degradation plots" graphs obtained in this experiment (Additional file 1) with those of 18 experiments stored in the PlexDB database from 2005 to 2013 (http://www.plexdb.org) [21] supports the high quality of the hybridizations reported in this work. The observed average background ranged from 37.4 to 46.0 units of expression, well within the parameters defined by Affymetrix, and comparable with the values obtained in other experiments that have used the GeneChip ${ }^{\circ}$ Wheat Genome Array [7,22]. The "present call" percentage ranged between $36.7 \%$ and $42.8 \%$ among the 61,000 probe sets indicating a degree of variability among the analyzed samples. The Pearson correlation was calculated between pairs of replications within each sample. The values ranged from 0.922 to 0.998 .

Four probe sets, representing genes putatively involved in the fatty acid metabolism (listed in Methods) were subjected to real-time GRT-PCR analysis to validate the microarray data. The observed Pearson correlation between microarray and qRT-PCR data was 0.753 (Additional file 2).

\section{Identification of differentially expressed genes}

The PCA (Principal Component Analysis) identified two principal components explaining $45.0 \%$ and $19.7 \%$ of the

Table 1 Relative Water Content (RWC) and leaf temperature of durum wheat plants subjected to drought, heat and to a combination of drought and heat stress

\begin{tabular}{llll}
\hline Genotype & Stress condition & RWC (\%) & Leaf temperature $\left({ }^{\circ} \mathbf{C}\right)$ \\
\hline Ofanto & Control & $96.97 \pm 1.47$ & $21.62 \pm 0.16$ \\
& Drought stress & $94.04 \pm 1.08$ & $21.70 \pm 0.07$ \\
& Heat stress & $85.96 \pm 2.02$ & $35.42 \pm 0.65$ \\
& Combined stress & $59.34 \pm 2.34$ & $38.36 \pm 0.98$ \\
Cappelli & Control & $95.86 \pm 1.06$ & $22.50 \pm 0.55$ \\
& Drought stress & $88.68 \pm 0.54$ & $22.00 \pm 0.10$ \\
& Heat stress & $82.88 \pm 0.45$ & $36.50 \pm 0.50$ \\
& Combined stress & $38.53 \pm 8.02$ & $41.00 \pm 0.20$ \\
\hline
\end{tabular}

RWC and temperature were determined on the leaf below flag leaf that was used for transcriptomic analysis. 
observed variability (Figure 1). The first component ( $x$ axis) accounts for the treatment effect, while along the second component (y-axis), the samples are separated according to the genotype. The vicinity between control samples (Ctrl) and drought stressed samples (DS) suggests a smaller effect of drought treatment compared to heat stress (HS) and to the combination of heat and drought stress (CS).

To identify the genes modulated in response to the applied stress conditions, five comparisons were carried out for each cultivar: Ctrl vs DS, Ctrl vs HS, Ctrl vs CS, DS vs CS and HS vs CS. Differentially expressed genes were identified based on Welch t-test with Benjamini and Hochberg correction for multiple testing [23]. A gene was considered differentially expressed when the $p$ value was less than 0.01 and the ratio of induction or repression was equal to or greater than 2 . The analysis yielded 4,212 not redundant differentially expressed probe sets (PSs) in Cappelli and 7,532 in Ofanto. Among these, 3,084 PSs differentially expressed were in common to both genotypes (Figure 2A). DS led to the modulation of 707 PSs in Ofanto and 248 in Cappelli with only 44 (27 up- and 17 down-regulated) in common (Figure 2B), while HS triggered the modulation of 3,243 PSs in Ofanto and 3,582 PSs in Cappelli with 1,402 PSs in common (Figure 2C). Moreover, a combination of drought and heat stress activated or repressed 5,645 PSs in Ofanto, and only 1,814 in Cappelli with 1,172 sequences in common (Figure 2D). Although the different number of PSs modulated in response to DS and HS can be due to different stress intensity (a more severe DS might lead to a higher number of differentially expressed genes), these results highlight very clearly that Cappelli and Ofanto have different stress response strategies with a minimal overlapping in terms of genes modulated in response to identical stress conditions. This finding was more evident in response to drought both as drought alone (DS) or in combination with heat (CS). In both conditions Ofanto modulated the expression of about three times more PSs than Cappelli.

The analysis of the 525 PSs up-regulated in response to DS in Ofanto and of their annotations highlighted that many of them were characterized by well-known drought-related functions. Furthermore, functional categories related to osmotic, salt, heat and cold response were over-represented among the annotation list. For

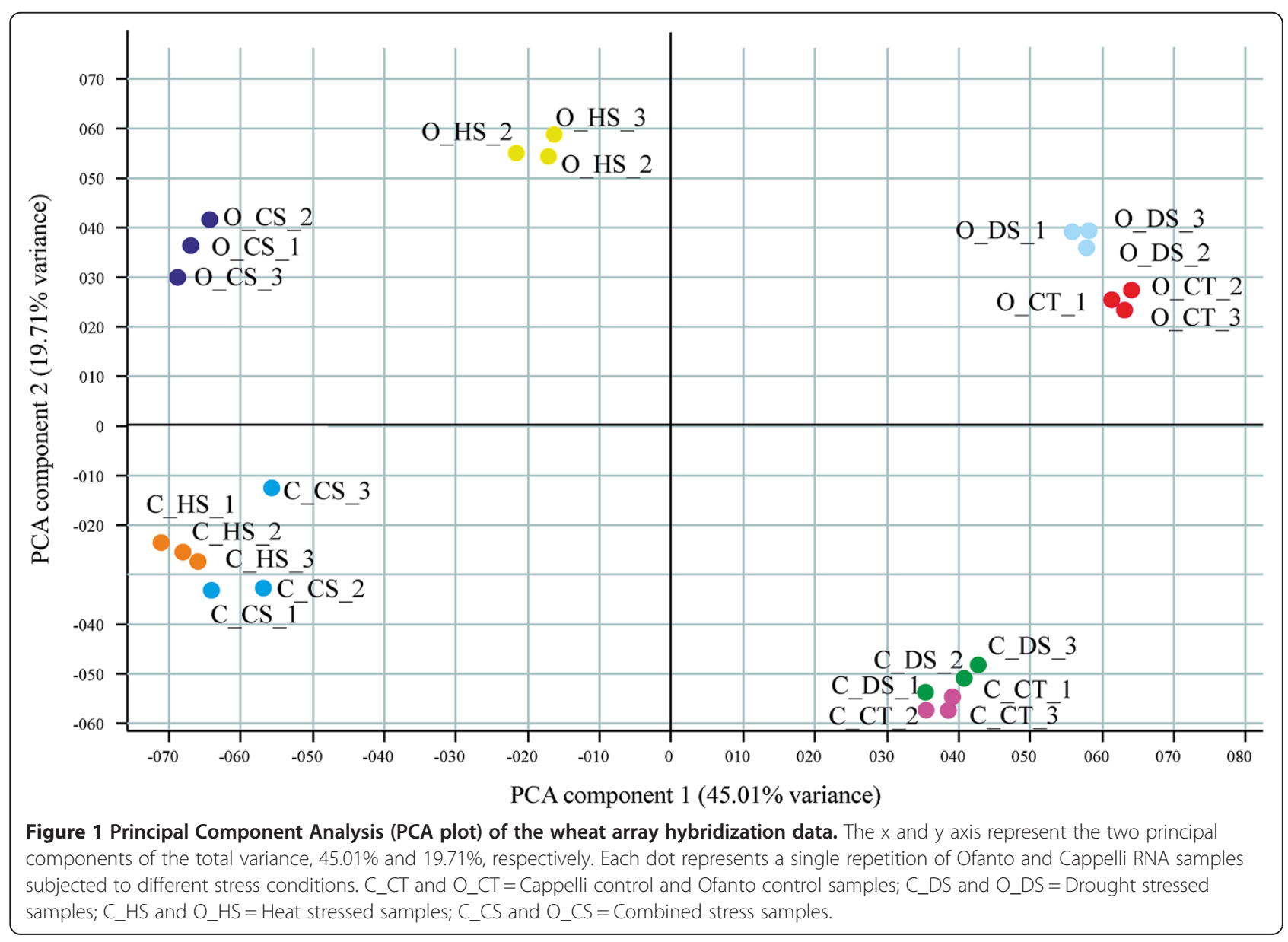




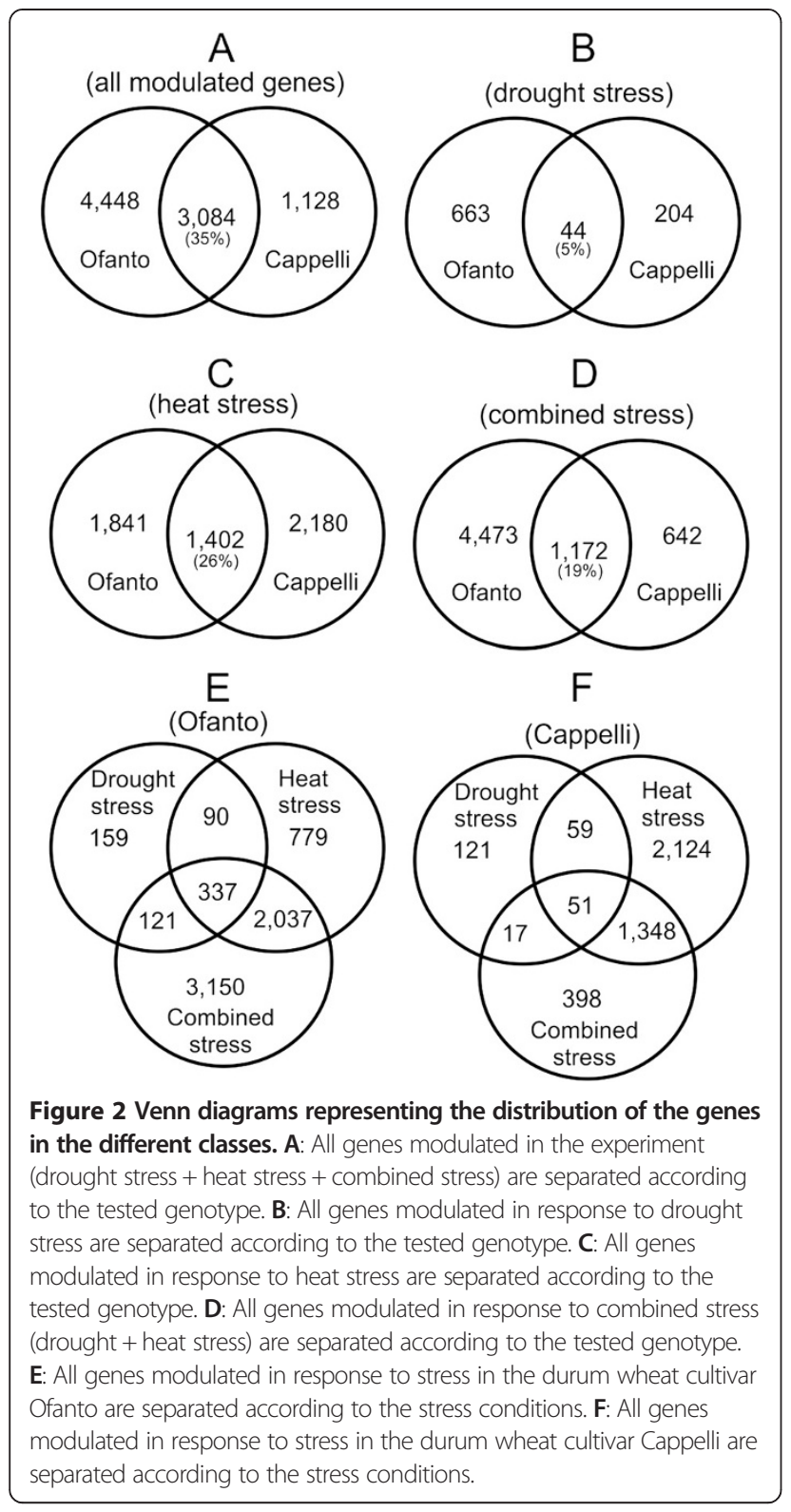

instance dehydrins (At1g01470, LEA14, [Ta.25026.1.S1_at]; At1g47128, RD21A, [Ta.5812.1.A1_at]; At4g39090, RD19A, [Ta.4419.2.S1_x_at]; At5g25610, RD22, [Ta.28209.3.S1_at, Ta.28209.3.S1_x_at]; At2g38905, Low temperature and salt response protein, [Ta.27725.1.S1_at]; At3g50970, LTI30/ Xero2, [TaAffx.131747.1.S1_x_at]; At4g36720, HvA22, [Ta.3038.2.S1_a_at]; At5g66400, RAB18, [10 different PSs, see Additional file 3]), lipid transfer proteins (At5g59310, LTP4, [Ta.1337.1.S1_x_at]; At5g59320, LTP3, [Ta.28368.2. S1_at]), heat shock proteins (At1g74310, HSP101, [TaAffx.56160.1.S1_at]; At3g09440, HSP70, [Ta.23807.1. S1_x_at]; At5g49910, CPHSC70-2, [TaAffx.114770.1.S1_at]), stress-related transcription factors (At4g38620, MYB4, [Ta.26049.1.S1_a_at]; At2g46270, GBF3, [Ta.13357.2.S1_at]), ABA and drought responsive proteins (At1g35720, ANN1,
[Ta.14590.1.S1_a_at]; At5g03280, EIN2, [Ta.7910.1.A1_at]; At2g26980, CIPK3, [TaAffx.106682.1.S1_at]) and genes coding proteins involved in drought responsive proline biosynthesis (At3g55610, P5CS, [Ta.7091.1.S1_at]; At5g14800, P5CR, [Ta.591.1.S1_at]) were all up-regulated by DS in Ofanto (Additional file 3). The identification of wellknown drought-related genes in Ofanto plants exposed to DS demonstrates that the stress conditions applied were effective in triggering a typical drought response in Ofanto, but not in Cappelli. This finding suggests, once again, a different perception of drought stress and, consequently, a different molecular response of the two cultivars.

Notably, 7 PSs corresponding to several genes (LEA14, ANN1, RD21A, HSP101, HSP70, CPHSP70-2, LTP4) induced by drought stress in Ofanto but not in Cappelli, showed a higher expression level in Cappelli Ctrl vs Ofanto Ctrl. These data suggest that a part of the genes expressed in Ofanto in response to drought were constitutively expressed in Cappelli. On the contrary, none of the 651 PSs expressed at higher level in Ofanto Ctrl $v s$ Cappelli Ctrl are known to be related to stress response.

In the cultivar Ofanto, CS treatment led to the modulation of about 5,645 PSs. Of these, 2,374 were in common with HS, 558 PSs were shared with the DS response and 3,150 PSs were specific of the combined treatment (Figure 2E). In Cappelli, CS modulated 1,814 PSs of which 68 were also modulated in response to DS and 1,399 in response to HS (Figure 2F). These results confirm previous findings [12-15] and highlight that the effect of CS is not a simple sum of drought and heat stress, rather it is perceived as a new stress condition.

\section{QT-clustering analysis identifies different expression profiles in Cappelli and Ofanto}

Besides the genes commonly regulated in the two cultivars, the stress response of Ofanto and Cappelli was characterized by significant differences that might be associated to the different physiological behavior of these genotypes under drought stress [17]. To highlight commonalities and differences between the two cultivars, a QT-cluster analysis was performed to identify groups of genes with an expression profile associated to specific or common responses in the tested genotypes. The QTclustering algorithm (Pearson correlation higher than 0.8 and minimum cluster size of 30 probe sets), run with 8,660 differentially expressed PSs, identified 52 clusters grouping 5,910 PSs plus 2,750 unclassified PSs (Additional file 4). Visual analysis of the 52 clusters highlighted a wide range of expression behaviors, no gene cluster was characterized by opposite trends between Ofanto and Cappelli (i.e. up-regulated in one cultivar and down-regulated in the other one). Some clusters showed an almost identical expression profile in the two cultivars (e.g. clusters 5, 13, 14, 21, 35 and 37 with 649 PSs in total), 
while the others highlighted some differences in gene expression between Ofanto and Cappelli.

Looking at the whole transcriptome numbers (Figure 2E and $2 \mathrm{~F}$ ) the most evident difference between the stress response of Ofanto and Cappelli was the much stronger response of Cappelli to heat stress. In this genotype 85\% of all PSs modulated in the experiment were responsive to HS (3,582 PSs out of 4,218; in Ofanto 49\%). Furthermore, the PSs regulated by HS and CS in Cappelli represent $77 \%$ of all PSs regulated in response to CS in this genotype (in Ofanto $36 \%$ ).

Some clusters highlighted PSs with a contrasting expression trend in the two cultivars across all treatments considered, these clusters could contain genes whose expression might contribute to explain the different molecular response of Ofanto and Cappelli to drought and heat stress. In this context the most relevant are the 9 clusters (namely clusters 1, 4, 8, 10, 17, 18, 23, 40 and
47) showed in Figure 3 and representing a total of 1,923 PSs, all up-regulated. In Cappelli, these genes were characterized by no or almost no response to DS, while, their expression in response to HS and CS was definitely enhanced. In Ofanto, the same clusters were up-regulated in at least one comparison among Ctrl $v s$ DS, Ctrl vs HS, Ctrl $v s$ CS. Overall the expression level of these genes was maximum under HS in Cappelli, while in Ofanto it was more elevated in response to CS. Since these clusters represent different gene regulation mechanisms, a specific data mining work has been undertaken to identify the key functions and pathways underlined by the selected clusters.

\section{Signaling components}

Among the 1,923 PSs belonging to clusters 1, 4, 8, 10, $17,18,23,40$ and 47 there were many components with a known role in the molecular events leading to stress tolerance (Table 2).

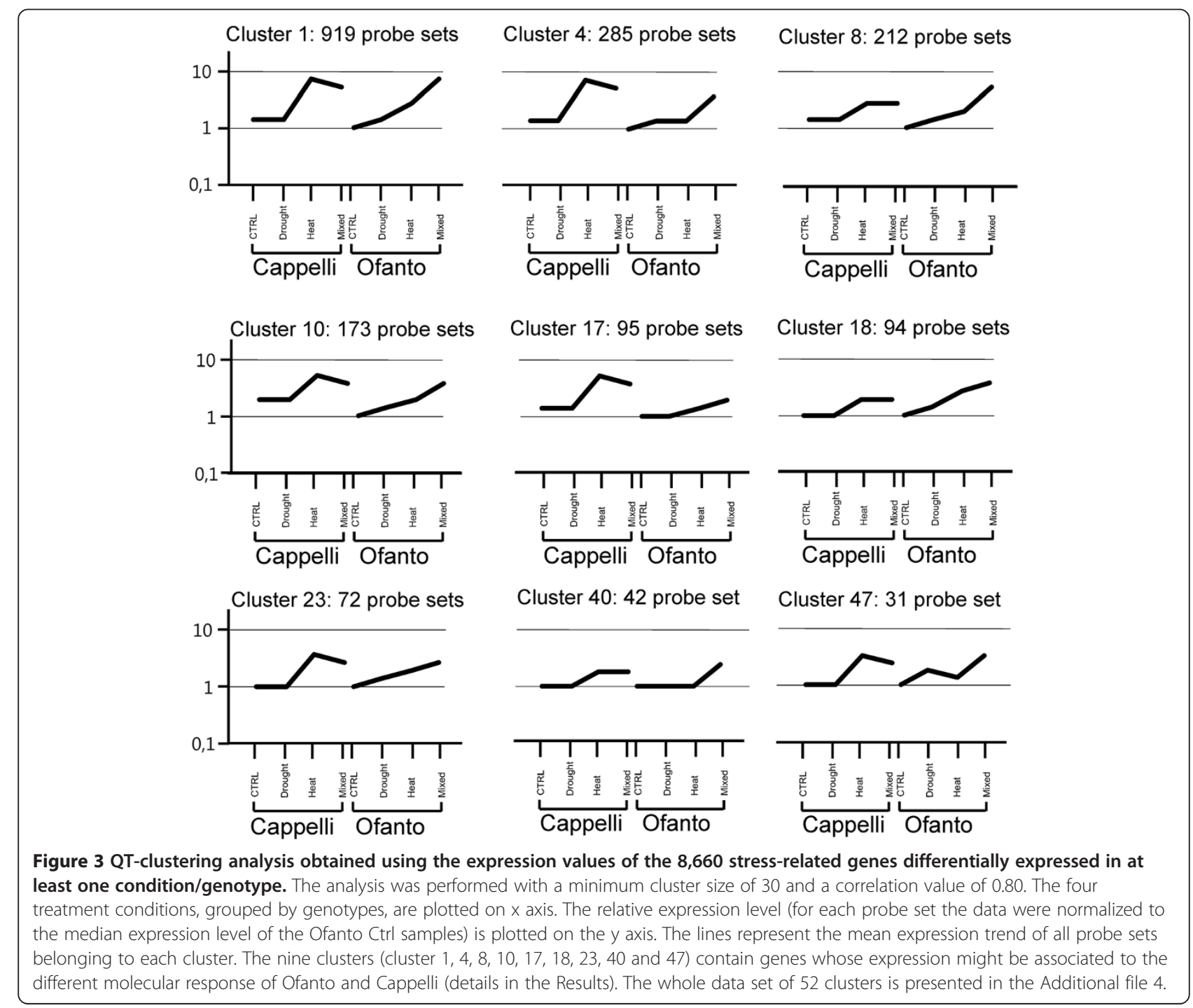


An early response to stress is represented by the activation of trans-membrane proteins acting as calcium transport channels resulting in a $\mathrm{Ca}^{2+}$ flow into the cytosolic region [24]. In Arabidopsis the calcium-binding RD20 protein (At2g33380) plays a key role in drought tolerance through stomatal control under water deficit condition [25]. On the GeneChip Wheat Genome Array six PSs with a high sequence similarity to the $R D 20$ gene are present, among them, one (Ta.9830.2.S1_at) was modulated by stress in the experiment here reported and exhibits an expression profile belonging to cluster 4 (Additional file 5). Its transcription was strongly activated in Cappelli in response to $\mathrm{HS}$ and CS, while in Ofanto it was activated only by CS (Additional file 5). Besides calcium binding activity, RD20 can play a role as peroxygenase in the oxylipin pathway [26]. Oxylipins are fatty acid hydroperoxide compounds active as germination inhibitors of fungal spores [27] and as modulators of plant development processes [28]. Nevertheless, when we analyzed the expression of all genes involved in the oxylipin pathway, no differentially expressed genes in response to stress were found, suggesting that the upregulation of $R D 20$, in our experiment, is not linked to oxylipin pathway regulation. Several other genes coding for $\mathrm{Ca}^{2+}$ binding proteins but without EF-hand motifs, like phospholipase D (PLD) were also found. The PLD catalyzes the cleavage of membrane phospholipids into phosphatidic acid (PA) and a soluble head group; its activity is regulated by $\left[\mathrm{Ca}^{2+}\right]$. PA, in turn, is a second messenger implicated in plant stress signaling [6], also produced by diacyl-glycerol kinase (DAG kinase) [29]. Two PSs (Ta.11144.1.A1_at, Ta.3908.1.S1_at) with high sequence similarity to Arabidopsis PLD (At3g15730) were differentially expressed with an expression profile following the one of cluster 1. Moreover two DAG kinase (At5g07920, At2g20900) related probe sets (Ta.3010.1. A1_at, Ta.3876.1.A1_at) belonging to clusters 4 and 8 (Additional file 5) were also differentially expressed.

Another key messenger in plants is the phosphatidylinositol (PI) that can be phosphorylated at several positions of the inositol head group. In the experiment here described, PI3K (At1g60490 [Ta.22091.1.A1_at, Ta.28795.1. S1_at]), PI(3,4)K (At1g13640 [TaAffx.110494.1.S1_x_at]) and PI4p5K (At4g33240 [Ta.5574.1.S1_a_at, TaAffx.86994.1. S1_at]; At3g14270 [Ta.5574.3.S1_at]) were differentially expressed and exhibited the same expression trend of cluster 1 , but for $\mathrm{PI}(3,4) \mathrm{K}$ belonging to cluster 23 . Notably, it has been reported that PI3K is required for ABA-induced ROS generation that leads to stomatal closure in Vicia faba [30]. Overall these data suggest that the calcium-related messengers and phosphoinositide signaling are involved in the stress response and that they are more active in Cappelli compared to Ofanto when exposed to HS (see the expression profiles of clusters 1 and 4, Figure 3).
An increased expression of genes encoding proteins involved in epigenetic regulation has also been associated to stress response. The variations of the chromatin structure are often dependent on the expression of histone variants or on histone post-translational modifications [31]. The drought, heat and combined stress activate the expression of different histone variants such as histone 4 (At5g59970 [Ta.10329.23.S1_at, Ta.10329.8.S1_x_at]), histone 1.2 (At2g30620 [Ta.23485.1.S1_at]), histone 2A (At4g27230 [Ta.644.1.S1_at], At1g54690 [Ta.6538.2.S1_at]), histone 2B (At2g37470 [Ta.7378.28.S1_x_at]), histone 3 (At5g10400 [Ta.27101.7.S1_at, Ta.27101.7.S1_x_at]), two histone deacetylases (At3g44680 [TaAffx.93321.1.S1_at], At5g61060 [Ta.1597.1.S1_at]) and a histone acetyltransferase (At5g64610 [Ta.5414.1.S1_at, Ta.5414.2.S1_a_at]), whose expression behaviors were associated mainly to cluster 1 , and to some extent also to clusters 4,8 and 40. Moreover two regulators of chromosome condensation, RCC1 (At3g02300 [Ta.15822.1.S1_at], At5g48330 [TaAffx.53414.1.S1_at], cluster 1 and 8, respectively) and a SMC2-like condensin (At5g62410 [Ta.20443.1.A1_at] cluster 17), were differentially expressed. Besides regulator of chromosome condensation genes, the WD-40 repeat protein (At5g58230 [Ta.13849.1.S1_at]), also named AtMSI1, was described to be involved in chromatin assembly [32]. The msi1 Arabidopsis mutant exhibits an enhanced expression of many ABA-responsive genes eliciting the response to drought and salt stress. Alexandre et al. [32] have demonstrated that MSI1 can bind to the chromatin of the drought-inducible downstream target RD20 suggesting for MSI1 a role in the negative regulation of Arabidopsis drought-stress response. However, in the durum wheat transcriptome analysis here reported, the upregulation of AtMSI1 was directly correlated to the expression of ABA-responsive genes and RD20. Fourteen probe sets with high sequence similarity to the gene coding the WD-40 repeat protein (clusters $1,4,8,10$, $17,18,23,40$ and 47) were found also by the present analysis (Additional file 5). Many of them are annotated as components of CUL4-based E3 ubiquitin ligases [33]. In particular At4g29380 [Ta.8384.1.S1_at] (also named AtVPS15) is a component of CUL4-based E3 ubiquitin ligases, but has a PI3K activity too, and is directly involved in PI3P production [34].

The analysis of the gene clusters differentially expressed between Ofanto and Cappelli during stress response identified also 21 probe sets related to proteins containing the RNA recognition motif (RRM) and splicing factors. These proteins were reported to be associated to the mRNA metabolism of genes involved in the ABA signal transduction in Arabidopsis [35] as well as in Vicia faba $[36,37]$. One of these proteins (AKIP1) is constitutively expressed in the nucleus of stomata guard cells, and it aggregates in small structures (speckles) involved in 
Table 2 Overview of the signalling component genes up-regulated by drought, heat and combined stress and belonging to cluster $1,4,8,10,17,18,23,40$ and 47

\begin{tabular}{|c|c|c|c|c|}
\hline Signaling component class & Gene name & AGI code & Probe set ID & Cluster \\
\hline $\mathrm{Ca}^{2+}$ binding protein & $R D 20$ & At2g33380 & Ta.9830.2.S1_at & 4 \\
\hline \multirow[t]{4}{*}{ Phosphatidic acid } & PLD & At3g15730 & Ta.11144.1.A1_at & 1 \\
\hline & & At3g15730 & Ta.3908.1.S1_at & 1 \\
\hline & DAG kinase & At5g07920 & Ta.3010.1.A1_at & 4 \\
\hline & & At2g20900 & Ta.3876.1.A1_at & 8 \\
\hline \multirow[t]{7}{*}{ Phosphatidylinositol } & PI3K & At1g60490 & Ta.22091.1.A1_at & 1 \\
\hline & & & Ta.28795.1.S1_at & 1 \\
\hline & $P I(3,4) K$ & At1g13640 & TaAffx.110494.1.S1_x_at & 1 \\
\hline & Pl4p5K & At4g33240 & Ta.5574.1.S1_a_at & 23 \\
\hline & & & TaAffx.86994.1.S1_at & 1 \\
\hline & & At3g14270 & Ta.5574.3.S1_at & 1 \\
\hline & AtVPS15 & At4g29380 & Ta.8384.1.S1_at & 8 \\
\hline \multirow[t]{16}{*}{ Chromatin condensation } & Histone 4 & At5g59970 & Ta.10329.23.S1_at & 1 \\
\hline & & & Ta.10329.8.S1_x_at & 1 \\
\hline & Histone 1.2 & At2g30620 & Ta.23485.1.S1_at & 1 \\
\hline & Histone $2 \mathrm{~A}$ & At4g27230 & Ta.644.1.S1_at & 1 \\
\hline & & At1g54690 & Ta.6538.2.S1_at & 4 \\
\hline & Histone $2 B$ & At2g37470 & Ta.7378.28.S1_x_at & 4 \\
\hline & Histone 3 & At5g10400 & Ta.27101.7_S1_at & 40 \\
\hline & & At5g10400 & Ta.27101.7_S1_xat & 40 \\
\hline & $H D A C$ & At3g44680 & TaAffx.93321.1.S1_at & 1 \\
\hline & & At5g61060 & Ta.1597.1.S1_at & 1 \\
\hline & HAT & At5g64610 & Ta.5414.1.S1_at & 1 \\
\hline & & & Ta.5414.2.S1_a_at & 8 \\
\hline & $\mathrm{RCCl}$ & At3g02300 & Ta.15822.1.S1_at & 1 \\
\hline & & At5g48330 & TaAffx.53414.1.S1_at & 8 \\
\hline & SMC2-like condensin & At5g62410 & Ta.20443.1.A1_at & 17 \\
\hline & AtMSI1 & At5g58230 & Ta.13849.1.S1_at & 1 \\
\hline \multirow[t]{5}{*}{ Stomatal closure } & OST1 & At4g33950 & Ta.991.1.S1_a_at & 1 \\
\hline & & & Ta.991.1.S1_at & 1 \\
\hline & & & Ta.991.1.S1_x_at & 1 \\
\hline & & & Ta.2551.1.S1_at & 4 \\
\hline & & & Ta.6040.1.S1_a_at & 18 \\
\hline \multirow[t]{9}{*}{ Temperature signaling } & $\mathrm{CHY}$ & At5g65940 & Ta.6750.2.S1_a_at & 1 \\
\hline & & & Ta.6750.3.S1_a_at & 1 \\
\hline & & & Ta.6750.3.S1_x_at & 1 \\
\hline & & & TaAffx.108337.1.S1_s_at & 1 \\
\hline & $B C K D H$ & At3g06850 & Ta.12252.1.S1_s_at & 4 \\
\hline & & & Ta.8293.1.A1_at & 4 \\
\hline & HSF & At3g22830 & Ta.28772.1.S1_at & 1 \\
\hline & & At2g26150 & TaAffx.105519.1.S1_at & 4 \\
\hline & HSPS & & 45 different probe sets ID & \\
\hline
\end{tabular}


mRNA storage or processing, in response to ABA. Moreover AKIP1 is activated through phosphorylation by AAPK (ABA Activated Protein Kinase) and, once activated, is able for example to bind dehydrin mRNAs [37]. In Arabidopsis the AAPK homolog gene (protein kinase OPEN stomata 1, OST1, At4g33950, also known as SnRK2E or $S n R K 2.6)$ is up-regulated by ABA and plays a key role in the phosphorylation events that lead to the ABA and osmotic induced stomata closure [38-41]. The wheat GeneChip ${ }^{\oplus}$ carries nine probe sets that have a sequence similarity with OST1. Of these, five were found to be up-regulated in durum wheat with an expression profile belonging to cluster 1 (Ta.991.1.S1_a_at, Ta.991.1.S1_at, and Ta.991.1.S1_x_at), cluster 4 (Ta.2551.1.S1_at) and cluster 18 (Ta.6040.1.S1_a_at). Another probe set, Ta.6918.1. S1_at, was identified in cluster 21 (Additional file 3).

$\mathrm{CHY}$ is a gene $(A t 5 g 65940)$ coding for a $\beta$-hydroxyisobutyryl CoA hydrolase (EC 3.1.2.4) involved in the catabolism of valine and with a role in the perception and transduction of low temperature signaling [42]. In durum wheat four probe sets (Ta.6750.2.S1_a_at, Ta.6750.3.S1_a_at, Ta.6750.3. S1_x_at, TaAffx.108337.1.S1_s_at) with high sequence similarity to the $C H Y$ were found in cluster 1 . A second gene involved in valine pathway and signal transduction was also differentially expressed; the branched chain alpha-keto acid dehydrogenase (BCKDH, EC 1.2.1.25) is localized in the mitochondria and catalyzes the reaction that leads to the synthesis of isobutyryl-CoA, a substrate of the reaction leading to hydroxyl-isobutyrate, catalyzed by $\mathrm{CHY}$ [43]. The wheat GeneChip ${ }^{\oplus}$ carries three probe sets with sequence similarity to $B C K D H$, two of them (Ta.12252.1.S1_s_at, Ta.8293.1.A1_at) were up-regulated according to cluster 4 expression profile.

The more pronounced response to HS of Cappelli vs Ofanto was also supported by two heat-shock factors $(H S F \mathbf{s},[44])$ related probe sets found in clusters 1 and 4, (At3g22830, Ta.28772.1.S1_at and At2g26150, TaAffx.105519.1.S1_at, respectively) whose expression promoted the up-regulation of $44 H S P$-related probe sets exhibiting a similar expression trend and belonging to clusters 1, 4, 8, 10, 17, 18, 23, 40 and 47.

Overall, the analysis of the genes belonging to clusters characterized by significantly different expression profiles between Cappelli and Ofanto allowed the identification of a number of key components of the heat and drought signaling pathways, suggesting that the different stress response of the two cultivars is supported by a different modulation of the regulatory mechanisms (Table 2).

\section{The fatty acids $\beta$-oxidation pathway}

In plants, the $\beta$-oxidation takes place mainly in peroxisomes and glyoxysomes. This pathway requires four enzymatic reactions that are repeated until the fatty acid degradation is completed [45]. The first reaction is catalyzed by acyl-CoA ligase/synthase (ACS, EC 6.2.1.3). There are twenty-two probe sets with high sequence similarity to the gene coding for this enzyme on the wheat GeneChip; seven were differentially expressed, among these four have expression trend according to cluster 17.

The activity of the acyl-CoA synthase leads to the formation of acyl-CoA that is converted to trans-2-enoylCoA by acyl-CoA oxidase (ACX, EC 1.3.3.6), the true first $\beta$-oxidation enzyme [46]. In the array there are 7 probe sets exhibiting sequence similarity to the Arabidopsis acyl-CoA oxidase genes (At5g65110, At3g51840, At1g06290), four of which were differentially expressed, three (Ta.10260.1.S1_at, Ta.9335.1.S1_at, Ta.9335.1.S1_s_at) belong to cluster 4, one (Ta.3420.2.S1_a_at) belongs to cluster 10. The previous reaction requires the presence of FAD as co-factor and $\mathrm{O}_{2}$ as electron acceptor; the latter is reduced to $\mathrm{H}_{2} \mathrm{O}_{2}$ that is subsequently decomposed by a peroxisome catalase (EC 1.11.1.16). Six peroxisome catalase-related probe sets were differentially expressed, four according to cluster 4 (Ta.1055.1.S1_at, Ta.1055.1.S1_x_at, Ta.1055.2.S1_at, Ta.1055.2.S1_x_at) and two according to cluster 12 (Additional file 3).

Subsequently, the trans-2-enoyl-CoA undergoes a hydration reaction catalyzed by enoyl-CoA hydratase $(\mathrm{ECH}$, EC 4.2.1.17) and it is transformed into the corresponding L- $\beta$-hydroxyacyl-CoA; in the next step, catalyzed by the L- $\beta$-hydroxyacyl-CoA dehydrogenase (HCD, EC 1.1.1.35), the L- $\beta$-hydroxyacyl-CoA is converted into $\beta$-ketoacyl-CoA, this transformation requires a molecule of $\mathrm{NAD}^{+}$that is reduced to NADH. In plant peroxisomes a multifunctional protein coded by At4g29010 is responsible for at least four activities, including the activity of enoyl-CoA hydratase and L- $\beta$-hydroxyacyl-CoA dehydrogenase [47]. Two wheat probe sets (Ta.9184.1.S1_at, Ta.2583.1. S1_x_at) have sequence similarity to the At4g29010 gene and they were both classified in cluster 4 .

The ending step of each $\beta$-oxidation cycle is the acetylCoA detachment from the starting fatty acid, catalyzed by the acyl-CoA-acetyltransferase enzyme or thiolase (AAT, EC 2.3.1.16). One probe set (Ta.28367.1.S1_at) has sequence similarity with the Arabidopsis thiolase gene and was induced by stress according to cluster 17.

In summary, all genes involved in the fatty acid $\beta$ oxidation, as well as a corollary gene of this cycle (peroxisome catalase), are activated by both HS and CS. The highest level of transcription was achieved by HS in Cappelli, while in Ofanto the level of transcription was much lower. The same pathway is activated in both cultivars under CS. A representation of the $\beta$-oxidation pathway with indicated variations in the expression levels of the genes coding for the key enzymes of the pathway is reported in Figure 4. 
The glyoxylate cycle

As $\beta$-oxidation pathway, also glyoxylate cycle occurs in peroxisomes and in glyoxysomes. In the glyoxysomes, acetyl-CoA is not further oxidized to produce energy and it is used as substrate to produce sugars. The reactions that occur in this pathway are known as glyoxylate cycle. In the glyoxysome, through a $\beta$-oxidation process, the fatty acids are converted into acetyl-CoA units and the reactions are catalyzed by well-known enzymes [48]. The expression level of the gene At3g16910 coding the enzyme named acyl-activating enzyme 7
(AAE7; EC 6.2.1.1), responsible for the acetyl-CoA synthesis, is described by four probe sets and, among these, one belongs to cluster 1 (TaAffx.31783.1.S1_at, Additional file 5) while the other three were not differentially expressed. The first step of the glyoxylate cycle is catalyzed by the citrate synthase enzyme (CS, EC 4.1.3.7). On the array there are three probe sets which show a sequence similarity with Arabidopsis citrate synthase gene (At2g42790) and, among these, one belongs to cluster 17 (Ta.970.2.S1_a_at) while the other two were not differentially expressed. Subsequently the

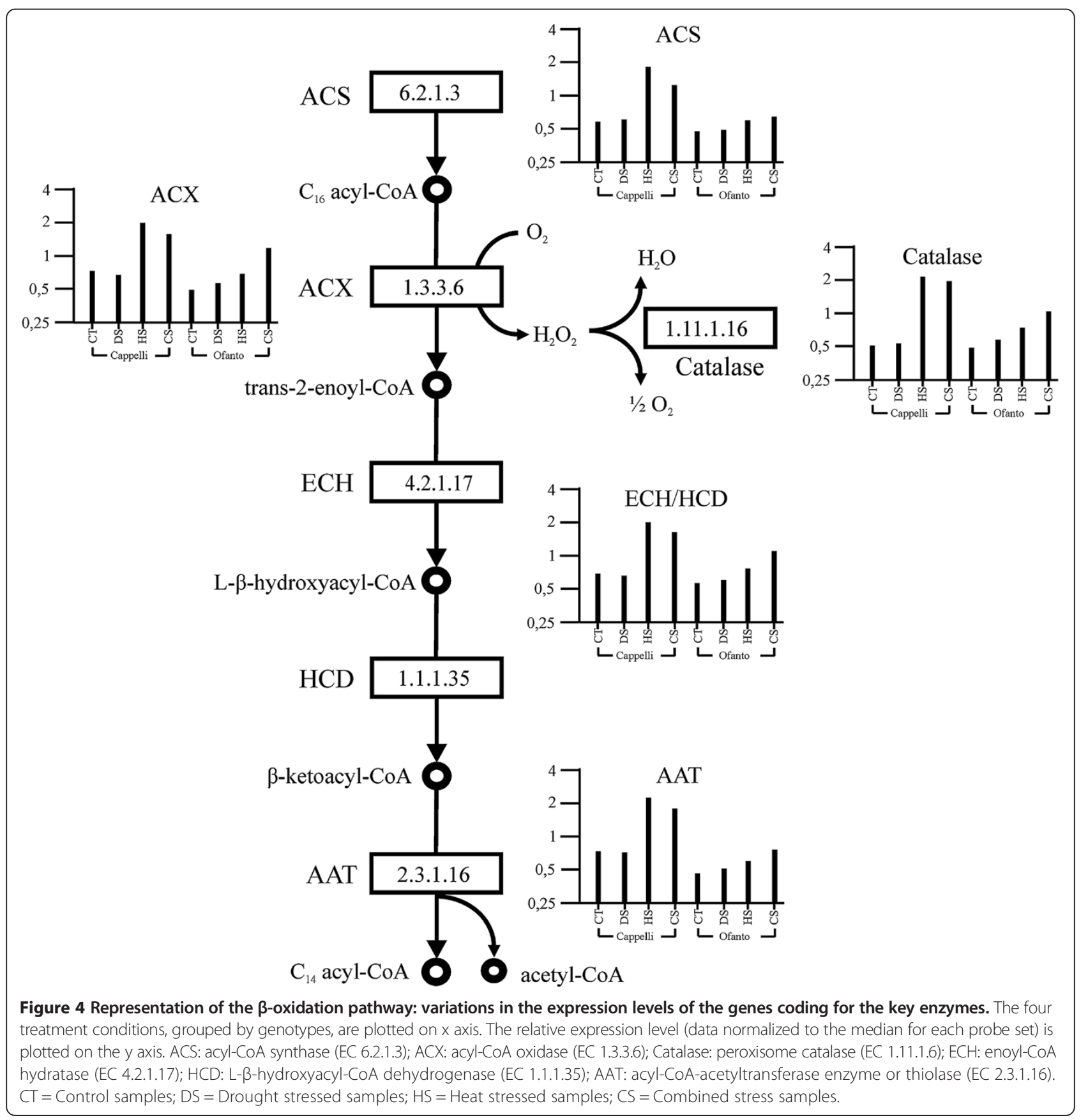


citrate is converted to isocitrate by a two-step reaction catalyzed by aconitase (ACO, EC 4.2.1.3). Among the twenty probe sets with high sequence similarity to aconitase genes, only one is differentially expressed (TaAffx.6397.1.S1_at) and belongs to cluster 30. The expression of the isocitrate lyase (ICL, EC 4.1.3.1) gene (At3g21720) responsible for the third step of the cycle is described by the TaAffx.79139.1.S1_at probe set that belongs to cluster 17. There are three other different probe sets that show high sequence similarity to At3g21720, but they were not differentially expressed. Five probe sets represent the gene $(A t 5 g 03860)$ for the malate syntase (MS, EC 2.3.3.9) that catalyze the fourth step of the cycle. Among these one (Ta.23970.1. A1_at) was differentially expressed and has been classified in cluster 17, another (Ta.23970.1.A1_x_at) has not been assigned to any cluster, and the remaining three were not differentially expressed (Additional file 3). The final step of glyoxylate cycle is catalyzed by malate dehydrogenase. In Arabidospsis there are 10 genes coding malate dehydrogenases (MDH, EC 1.1.1.37) that act in cytoplasm, mithocondria, chloroplasts and glyoxysomes. The wheat GeneChip ${ }^{\circ}$ carries 48 probe sets and only one of them is differentially expressed (Ta.25543.2. S1_at) and belongs to cluster 17 .

Similarly to the fatty acid $\beta$-oxidation pathway, the glyoxylate cycle appear to be activated by HS specifically in Cappelli, and by CS in both genotypes. A representation of the glyoxylate cycle illustrating the variations in the expression levels of the genes coding for the key enzymes of the pathway is reported in Figure 5.

\section{Senescence marker genes}

The activation of the glyoxylate cycle genes is considered a clear sign of plant cell senescence $[49,50]$. To study putative correlation among glyoxylate cycle activation and senescence in durum wheat, several genes, known as senescence markers [51], were checked. A NAC transcription factor (At5g39610, NAC6/ANAC092) was already described as a gene involved in salt promoted senescence [52] and in this experiment it has a cluster 26 expression trend. In Arabidopsis the gene At3g56400 (WRKY70) is a negative regulator of senescence [53]. The wheat GeneChip ${ }^{\circ}$ has three probe sets with high sequence similarity to WRKY7O and one of them was down-regulated with an opposite expression trend if compared to glyoxylate cycle genes. A third transcription factor involved in senescence processes (TaNAM-B1) was found differentially expressed and belongs to cluster 4. TaNAM-B1 and its homologs are responsible for senescence processes in wheat [54].

Although our data do not show a wide activation of senescence-related genes, several senescence transcription factors were clearly activated with an expression trend correlated to glyoxylate cycle and to many stress response genes, indicating the activation of early heat stress-induced senescence in Cappelli.

\section{Modulation of gene expression induced by drought at tillering stage and e-QTL mapping}

The comparisons of the transcriptomic data as well as the QT-clustering experiment have indicated a different strategy in the response of Cappelli and Ofanto to drought and heat stress at booting stage. Since the difference in WUE between the two cultivars is manifested constitutively through all life cycle [17], 15 drought responsive genes at booting stage (10 more expressed in Ofanto DS vs Cappelli DS and 5 vice-versa) were also tested in drought treated plants at tillering stage. All genes confirmed their drought responsiveness in one or in both cultivars. In each cultivar, 12 genes induced by drought were found. The comparison of the expression level of the genes in Ofanto drought vs Cappelli drought highlighted 8 genes with a significantly higher expression level in Ofanto $v s$ Cappelli (fold change $>2$ ), while the remaining 7 genes, although generally more expressed in Ofanto, were not significantly different (Figure 6, Additional file 6). These findings suggest that at tillering stage the response of Ofanto to drought is stronger, in terms of gene expression levels, than the response of Cappelli to the same condition. This results, once again, highlights the different response strategy of these two cultivars to drought conditions with a stronger molecular response in Ofanto that is, to some extent, coherent with the results of the transcriptomic analysis performed at booting stage.

To gain more details on these contrasting responses we have taken advantage of the genetic tools available for this couple of genotypes and we tested the possibility of mapping QTLs controlling the expression level (e-QTLs) of genes differentially expressed between the cultivars tested. The experiment was carried out using the gene corresponding to the probe set TaAffx.100436.1.S1_at (annotated as High Expression Level gene, HEL). At booting stage, the microarray data indicate that this gene was expressed in Ofanto and up-regulated in response to all stress treatments, while an expression below the background value was detected in Cappelli in all tested conditions (Additional file 3). When the expression profile of $H E L$ has been verified in an independent experiment carried out at tillering stage in plants subjected to severe drought stress (27-28\% RSWC), the qRT-PCR analysis have revealed that HEL is clearly expressed in both cultivar in the absence of stress (Ctrl) and down-regulated in response to drought treatment. Nevertheless, the HEL transcription level resulted to be much lower in Cappelli than in Ofanto (Additional file 6), so that the fold change 


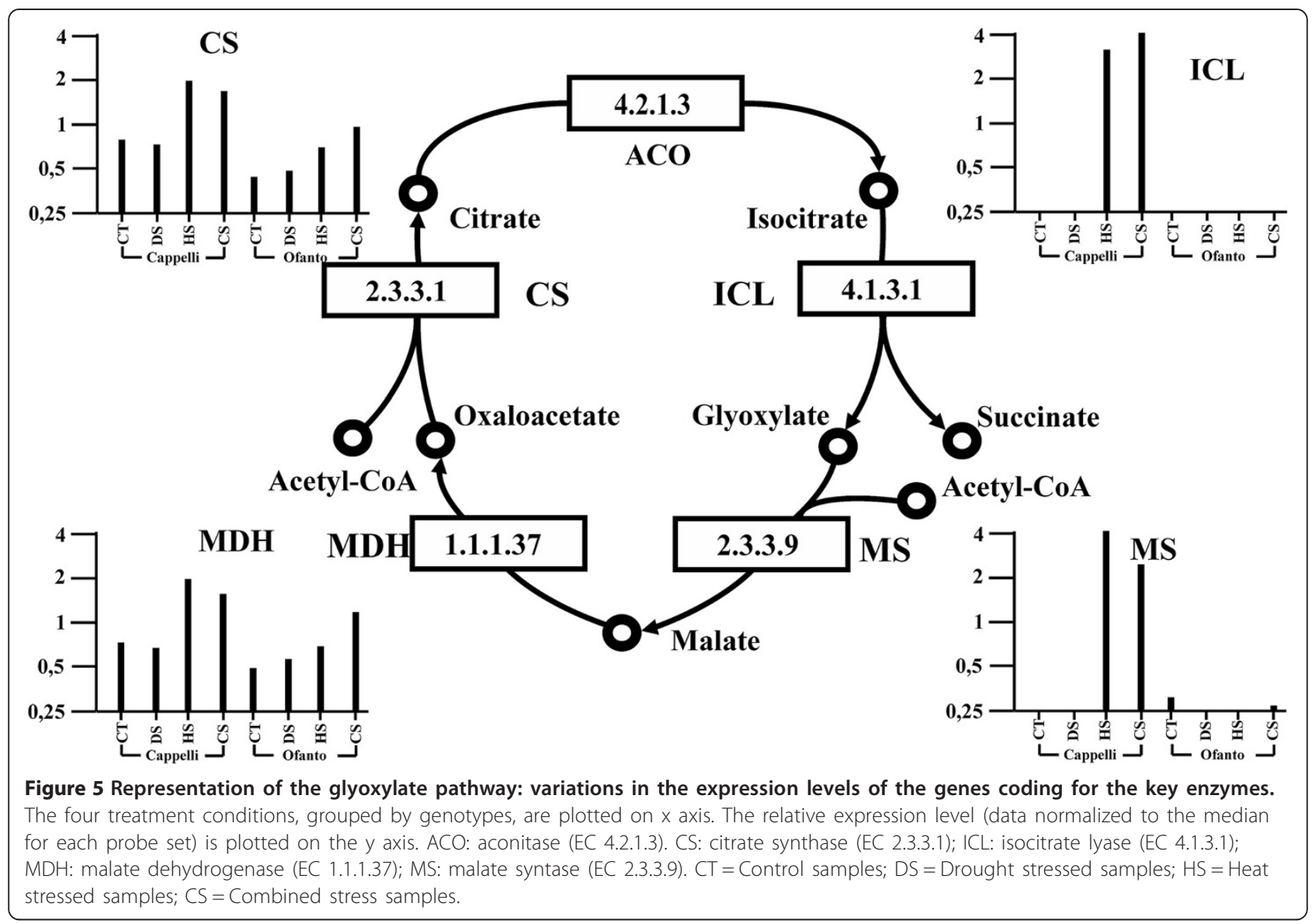

value of the ratio Ofanto drought vs Cappelli drought was 299-fold higher in Ofanto with respect to Cappelli (Figure 6). This great difference in gene expression associated to drought response was considered an ideal requirement to identify e-QTLs controlling drought gene expression.

The sequence of HEL transcript was obtained after "blastn" submission of the TaAffx.100436.1.S1_at probe set in CerealsDB database (Chinese spring Contig444272, 1,448 bp), TIGR Plant Transcript Assemblies (TA97230_4565) and DFCI Wheat Gene Index database (TC419595). A research for ortholog genome sequences in close related Brachypodium and Oryza species, a search for coding region detection in DNA sequences using ESTScan as well as an analysis for protein sequence in UniProt did not provide any information about these sequences annotation and function. Otherwise, alignment of these sequences detected a putative intron (Additional file 7).

The accumulation of HEL transcripts in response to drought was tested on 80 Recombinant Inbred Lines (RILs) from the cross Ofanto $x$ Cappelli exposed to drought at tillering stage. The expression data showed a bimodal frequency distribution in the RIL population that indicates a simple genetic control of the trait (Figure 7). When the Ofanto $\mathrm{x}$ Cappelli genetic map, also including the marker corresponding to the HEL gene, was used to perform the e-QTL analysis, a single e-QTL with a strong effect located in the telomeric region of the long arm of chromosome 6B was detected. The e-QTL showed a LOD of 43.9 , with $95.2 \%$ of the observed variability explained, and a negative additive effect was found (-4.93), which indicated that the allele carried by Ofanto was effective in increasing the HEL expression level. Notably, the e-QTL was positioned in the same chromosomal region where the HEL gene was mapped (Figure 7), a finding suggesting that the main factor controlling HEL expression might rely in the gene sequence itself.

\section{Conclusions}

Ofanto and Cappelli: two different stress responsive strategies

The durum wheat cultivars Ofanto and Cappelli represent an ideal experimental system to investigate the water stress response in durum wheat. The two cultivars are characterized by a significantly different WUE [16,17], an Ofanto x Cappelli RIL population has been implemented with a molecular marker map $[19,20]$ and it is currently used to map some WUE-related traits [18]. 


\section{Ofanto drought vs Cappelli drought - 27/28\% RSWC}

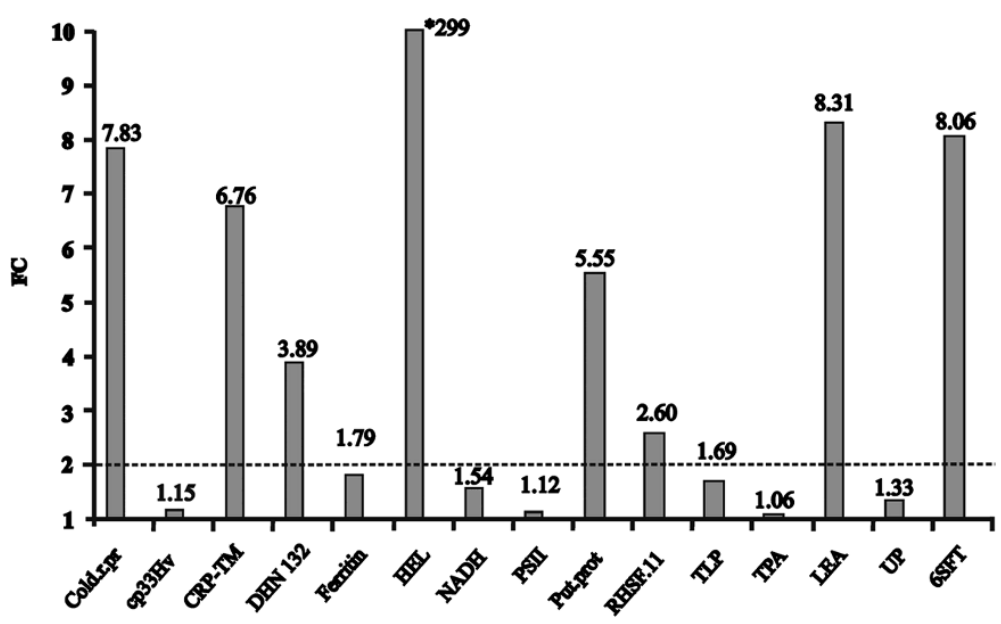

Figure 6 Fold Change values (FC, number on each column) of the expressed genes selected based on the array hybridization data of flag leaves at booting stage and tested in leaves of drought treated young plants at tillering stage by qRT-PCR analysis. The FC value is the ratio of the expression level of the selected genes in the comparison Ofanto vs Cappelli samples subjected to severe drought stress of 27/28\% Relative Soil Water Content (RSWC). The acronyms of the genes in the figure mean: Cold.r.pr (Cold regulated protein, Ta.13183.1.S1_x_at); Cp33Hv (RNA-binding protein cp33 precursor-barley, Ta.18434.1.S1_at); CRP-TM (cold regulated protein, Ta.13183.1.S1_s_at); DHN 132 (dehydrin, DHN, Ta.13255.1. S1_at); Ferritin (Ferritin, Ta.681.2.S1_a_at); HEL (High Expression Level; TaAffx.100436.1.S1_at); NADH (NADH dehydrogenase subunit B, TaAffx.112816.1.S1_at); PSII (Photosystem II 10 K protein precursor; Ta.28750.2.A1_x_at ); Put. Prot (Putative protein, Ta.29464.1.A1_at); RHSF.11 (heat shock factor RHSF11, TaAffx.34778.1.S1_at); TLP (thaumatin-like protein, Ta.25053.1.S1_at); TPA (transposase, Ta.3145.1.S1_at); LEA (Late Embryogenesis Abundant protein, Ta.5913.1.S1_at); UP (Unknown Protein, Ta.28273.1.S1_x_at); 6-SFT (Sucrose:fructan 6-fructosyltransferase, Ta.2789.2.S1_at). * this peak was corrected for comparison to the others.

The present work was designed to provide an additional level of knowledge on the Ofanto and Cappelli experimental system through a transcriptomic analysis of the molecular response to drought, heat, and a combination of both stress conditions.

The transcriptomic data highlight that Ofanto activated a large set of well-known drought-related genes after drought treatment, while Cappelli showed the constitutive expression of several genes that in Ofanto are induced by drought and a minimal modulation of gene expression in response to stress. Assuming that the extent of gene modulation (number of genes modulated in response to stress) is a consequence of the stress signal perception, the same experimental conditions, determined as SWC, had a different impact both on RWC and on stress signaling in Cappelli and Ofanto. Despite the lower RWC of Cappelli compared to Ofanto, the former cultivar showed a minimal gene activation in response to drought. The lower stomata conductance [17] and the constitutive expression of some drought-related genes might contribute to limit the effect of drought and the stress perception in Cappelli which, in turn, is reflected in a minimal drought-induced gene expression.

The heat response was characterized by the remarkable induction, in Cappelli only, of the genes coding for the enzymes involved in the fatty acids $\beta$-oxidation and in the glyoxylate cycle. An enzyme of the $\beta$-oxidation pathway, the thiolase (AAT, EC 2.3.1.16), is also involved in ABA signaling. Jiang et al. [55] have provided genetic evidence that thiolase positively regulates $\mathrm{ABA}$ signaling, including ABA-induced stomata closure in Arabidopsis. Overexpression of the gene coding thiolase increased biosynthesis of jasmonic acid (JA) and accelerated dark-induced senescence of leaves; the opposite characteristics were observed in antisense transgenic lines [56]. The enhanced expression of the thiolase gene, together with the activation of the glyoxylate cycle genes (generally associated with senescence $[49,50])$ and with the modulation of some senescence marker genes, might suggest an early activation of the senescence and of senescence-related processes in Cappelli but not in Ofanto.

The combination of drought and heat stress in plants is a unique stress sharing a marginal portion of the molecular responses activated by drought and heat stress alone [12-15,57-59]. This is evident in the case of the cultivar Ofanto that activates 707 PSs in response to drought stress, 3,243 in response to heat stress and 5,645 in response to drought and heat combined stress. However we observed the opposite phenomenon in Cappelli, since it modulates 248 PSs in response to drought stress, 3,852 PSs in response to heat stress and "only" 1,814 in response to the drought and heat combined stress. 


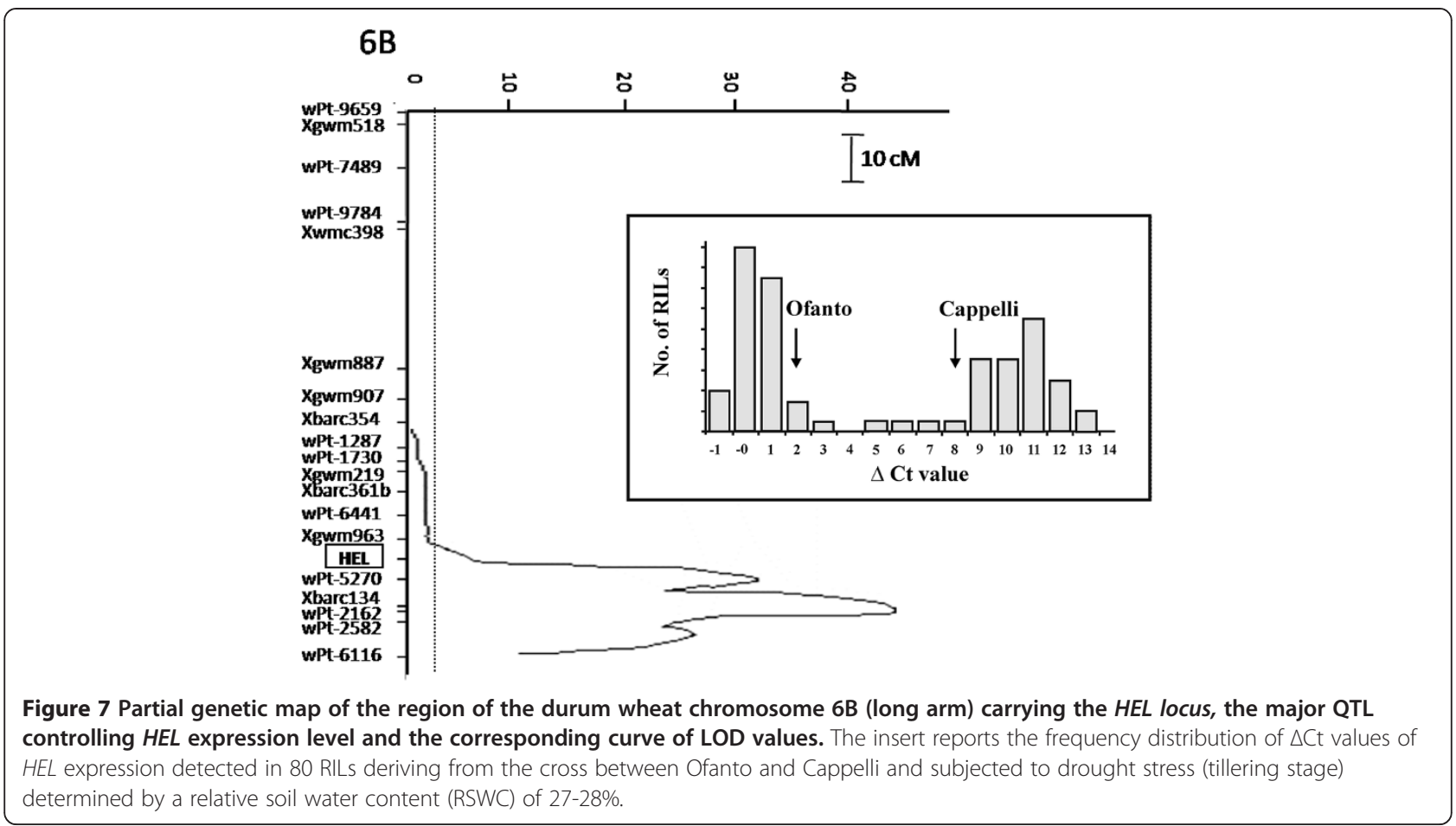

The great differences found in the stress response between Ofanto and Cappelli at transcriptomic level underline that cultivars of the same species can use different molecular strategies to cope with unfavorable climatic conditions. Other examples are also known; the different level of salt-tolerance between FL478 (tolerant) and IR29 (sensitive) rice varieties was associated to a different expression of transcripts corresponding to cation transport proteins, involved in reducing $\mathrm{Na}^{+}$influx, and enzymes with antioxidant activity as peroxidases [60,61]; the different heat tolerance of TAM107 (tolerant) and Chinese Spring (sensitive) bread wheat cultivars was associated to 313 genes differentially expressed between the two varieties in response to heat stress [10].

\section{e-QTL analysis in young plants}

Many studies are available in literature about linkage mapping of genomic regions involved in the control of agronomic traits, but few are still the evidences about e-QTLs [62-64]. A pioneering study in this field was represented by the e-QTL analysis of Cor14, a cold regulated gene of wheat and barley. Cor14 gene expression was higher in frost resistant vs susceptible cultivars and the QTL analysis identified a single e-QTL co-segregating with a frost tolerance QTL and with the CBF locus, coding key $\mathrm{CBF}$ transcription factors controlling the cold response $[65,66]$. In this study a similar approach was carried out with a drought induced gene (HEL) showing a marked difference in expression level between Ofanto and Cappelli exposed to drought. The expression of a gene can be controlled by a number of different factors, from epigenetic to translational level. In general, cisacting factors, like features of promoters or introns as examples, or trans-acting factors, as transcriptional regulators or protein acting on mRNA splicing, translation or stability of transcripts, can influence the accumulation of the mRNA of a gene [67]. The fact that the analysis of $H E L$ gene expression has revealed a single e-QTL with a strong effect (more than $90 \%$ of explained phenotypic variability) coincident with the HEL locus strongly suggests that the main factor controlling its expression relies in the gene sequence itself. Otherwise, if a transacting factor is somehow involved, we can conclude that it is located in the same genomic locus of the HEL gene. However, the transcription profiles of 41 double haploid wheat lines using the Affymetrix GeneChip Wheat Genome Array have revealed the putative position of 542 genes identified as major e-QTLs on single-chromosomal regions [68].

Some QTLs for traits related to grain yield in dry environments were previously identified in the region of the e-QTL found in this study, based on molecular markers in common with published genetic maps [69,70]. Since large parts of the wheat genome are covered with QTLs for drought-related traits, a genetic association between a QTL and an e-QTL is not sufficient to infer for a role of the gene in drought tolerance, but it can represent an indication to be tested with further experiments.

The mapping of the e-QTL controlling HEL gene expression, together with other experiments here reported, 
provides clear evidences that the genetic system based on Cappelli and Ofanto represents an ideal tool for the genetic dissection of the molecular response to drought and other abiotic stresses.

\section{Methods}

Plant material and growth conditions

For transcriptomic analysis at booting stage two Triticum turgidum subsp. durum cultivars, Ofanto and Cappelli, were grown in a growth chamber under conditions mimicking the natural growing season. Germination was carried out at $6^{\circ} \mathrm{C}$ in complete darkness until shoot emergence, then the growth conditions were set at $10 \mathrm{hrs}$ light and $14 \mathrm{hrs}$ dark at $8^{\circ} \mathrm{C}$ and $6^{\circ} \mathrm{C}$, respectively, relative humidity $60 \%$. Growing conditions were then modified according to the plants growth stage: $13 \mathrm{hrs}$ light and 11 hrs dark with $12^{\circ} \mathrm{C}$ and $8^{\circ} \mathrm{C}$, respectively, relative humidity $50 \%$ at second leaf stage; 14 hrs light and $10 \mathrm{hrs}$ dark with $15^{\circ} \mathrm{C}$ and $10^{\circ} \mathrm{C}$, respectively, relative humidity $50 \%$, at the third leaf stage; 15 hrs light and 9 hrs dark with $15^{\circ} \mathrm{C}$ and $12^{\circ} \mathrm{C}$, respectively, relative humidity 50\%, at tillering stage; 15 hrs light and 9 hrs dark with $19^{\circ} \mathrm{C}$ and $15^{\circ} \mathrm{C}$, respectively, relative humidity $40 \%$ at booting stage. Light was provided by $400-\mathrm{W}$ high-pressure lamps (Philips) with a photon flux density of $500 \mu \mathrm{mol} \mathrm{m}^{-2} \mathrm{~s}^{-1}$.

Plants were grown in pots $(38 \times 16 \mathrm{~cm}, 14 \mathrm{~cm}$ depth, 12 seeds/pot) filled with a substrate of soil (clay-loam), sand and peat in a 6:3:1 ratio, respectively. Pots filled with the substrate were standardized for weight and field capacity. A complete randomized block design with three replications was adopted. Ammonium biphosphate (presowing) and ammonium nitrate (shoot emergence and tillering) were used as fertilizers for a total amount equivalent to $60 \mathrm{~kg} / \mathrm{ha}$ of P and $150 \mathrm{~kg} / \mathrm{ha}$ of N. Powdery mildew was controlled with fungicide (Folicur $1.5 \mathrm{~g} / \mathrm{L}$ ) from tillering onwards. The irrigation was made with tap water ( $0.5 \mathrm{dS} \mathrm{m}-1)$, weighting pots and intervening whenever the soil lost $50 \%$ of available water, bringing it back to a soil water content (SWC) corresponding to $28 \%$.

Drought stress (DS), heat stress (HS) and combined stress (CS) were imposed simultaneously when the plants reached the booting stage. Control (Ctrl) plants were kept in the conditions described above and watered to maintain SWC equal to $28 \%$. DS was imposed by withholding water and allowing the pots to reach SWC equal to $12.5 \%$. To monitor SWC the pots weight was measured twice per day. The flag leaves detached at mid of the light period were collected for transcriptomic analysis three days after the imposition of stress. HS was imposed by increasing the temperature for two days at $30^{\circ} \mathrm{C}$ and $22^{\circ} \mathrm{C}$ in the $15 \mathrm{hrs}$ light and $9 \mathrm{hrs}$ dark regime, for an initial adaptation phase, then the temperature was increased to $34^{\circ} \mathrm{C}$ and $26^{\circ} \mathrm{C}$ light/dark for two days and subsequently to $40^{\circ} \mathrm{C}$ and $32^{\circ} \mathrm{C}$ light/dark for an additional day when the flag leaves were collected. CS was imposed withholding water to reach SWC equal to $12.5 \%$ as described for DS, and increasing the temperature up to $40^{\circ} \mathrm{C}$ as described for HS. Before leaf sampling, the flag leaf temperature was evaluated using the Testo-925 thermometer (Table 1). The plant water status was monitored as Relative Water Content (RWC\%) determined on the same plants used for RNA isolation using the leaf below the flag leaf (Table 1). All experiments were carried out in triplicate. Leaf material used for molecular analysis was immediately frozen in liquid nitrogen and kept at $-80^{\circ} \mathrm{C}$.

An additional transcriptomic analysis was carried out at tillering stage. Seedlings of Ofanto, Cappelli and 80 Recombinant Inbreed Lines (RILs) from the cross Ofanto $x$ Cappelli were transferred to pots of 9.5, 7.0 and $17 \mathrm{~cm}$, top diameter, bottom diameter, and depth, respectively (one plant for each pot) at Zadoks stage 0.7 [71], i.e. when the coleoptile had emerged from the caryopsis. The plants were grown in a growth chamber, at $13 \mathrm{hrs}$ light and $11 \mathrm{hrs}$ dark at $20^{\circ} \mathrm{C}$ and $15^{\circ} \mathrm{C}$ respectively, with average quantum flux density of PAR of $400 \mu \mathrm{mol} \mathrm{m}^{-2} \mathrm{sec}^{-1}$ and humidity of $70 \%$ (day) and $90 \%$ (night). Before planting, the soil dry weight (DW) and the weight of soil watered till field capacity (WFC) were measured. Relative soil water content $(\mathrm{RSWC})$ was calculated as: $\mathrm{RSWC}=$ ((current pot weight - DW)/(WFC-DW))*100. A slow water stress was applied by withholding water for 23 days starting at stage DC14-19 (Zadoks scale) and approximately till tillering (Zadoks stage DC22-29) when the final RSWC level was $27-28 \%$ which corresponds to a severe stress. Pots were weighted daily and control plants were watered to $95 \%$ of RSWC continuously. The uppermost fully expanded leaves were harvested all at the same time and used for RNA isolation.

\section{RNA isolation and array hybridization}

Total RNA was extracted from leaf tissues by TRIZOL reagent according to the method published by the Arabidopsis Functional Genomics Consortium http://www.arabidopsis. org/portals/masc/AFGC/RevisedAFGC/site2RnaL.htm\# isolation. RNA was cleaned using RNeasy columns according to the Qiagen RNeasy Mini Handbook. To assess RNA quality and quantity, several dilutions of each sample were analysed using the Agilent RNA 6000 nano Kit and Agilent Bioanalyzer 2100. RNA samples were processed following the Affymetrix GeneChip Expression Analysis Technical Manual (Affymetrix). Single-stranded, then double-stranded cDNAs were synthesized from the poly(A) mRNA isolated from $5 \mu \mathrm{g}$ of total RNA for each sample using the Affymetrix One-Cycle Labeling kit and Control reagents. The resulting $d s-\mathrm{cDNA}$ was column purified and then 
used as a template to generate biotin tagged cRNA from an in vitro transcription reaction (IVT), using the Affymetrix GeneChip IVT Labelling Kit. Fifteen $\mu \mathrm{g}$ of the resulting biotin-tagged cRNA was fragmented to strands of 35-200 bases in length following prescribed protocols (Affymetrix GeneChip Expression Analysis Technical Manual) and then hybridized at $45^{\circ} \mathrm{C}$ with rotation for 16 h (Affymetrix GeneChip ${ }^{\circ}$ Hybridization Oven 640) to probe sets present on an Affymetrix GeneChip Wheat Genome Array. The arrays were washed and then stained (SAPE, Streptavidin-phycoerythrin) on an Affymetrix Fluidics Station 450 followed by scanning with a GeneChip ${ }^{\circ}$ Scanner 3000. Wheat microarray design and expression profiling data are available in PlexDB (http://www.plexdb.org) [72] as experiment "TA47" and at http://www.ncbi.nlm.nih.gov/geo as experiment GSE45563.

\section{Data processing and analysis}

GeneChip hybridization quality was ensured using the standard Affymetrix controls. B2 oligonucleotides were spiked into each hybridization cocktail. PolyA controls (lys, phe, thr, dap) and hybridization controls (BioB, BioC, $B i o D$ and $\mathrm{Cre}$ ) were used to monitor the labelling and hybridization processes. To draw the "RNA degradation plot" relative to probe signal intensities of the actin and GAPDH control genes, the R "simpleaffy" library was used. Raw intensity values were normalized by RMA (Robust Multi-array Average) [73] using the R package "Affy" [74]. The same package was used to run the MAS 5.0 algorithm on raw data to produce a detection call for each probe set. These detection calls ("present", "marginal" or "absent") were used to apply an initial filtering step, since genes not expressed ("absent") represent experimental noise and can generate false positives. We removed from analysis all the probe sets that did not show all the three "present" calls in at least one sample. R-squared linear correlation coefficients were computed on the RMA expression values (log2-transformed) for each set of biological triplicates. RMA filtered data were imported to the software Genespring GX 7.3 (Agilent Technologies) and all subsequent analyses were carried out with this software.

Differentially expressed probe sets were identified through a Welch t-test with Benjamini and Hochberg false discovery rate correction for multiple tests [23]. Differences in gene expression were considered to be significant when $\mathrm{p}$-value was lower than 0.01 and induction or repression ratio was equal or higher than 2-fold. Principal Component Analysis (PCA, [75]) was employed to assess the contribution of genotype and treatment factors in the variation detected in the dataset. Clusters of genes with distinctive expression patterns were searched with QT (Quality Threshold) cluster analysis [76]. QT clustering algorithm groups genes into high quality clusters based on two parameters: "minimum cluster size" and "minimum correlation". The minimum cluster size was set to 30 and minimum correlation to 0.80 . Functional gene categories over-represented in the clusters in comparison with the whole microarray were searched at the MIPS Arabidopsis thaliana database (MAtDB) Functional Catalogue (FunCat, http://mips.helmotz-muenchen.de/proj/funcatDB). MIPS FunCat is a hierarchical database that links Arabidopsis locus identifiers to functional categories. The FunCat database currently contains 28 main categories subdivided into 1,289 subcategories [77]. Blast searches were done using HarvEST: Affymetrix Wheat1 Chip 1.50 (http://www.harvest.ucr.edu) and only the annotations of wheat probe sets with a homology level cut-off equal or lower than $\mathrm{E}$-value $=\mathrm{e}^{-10}$ were considered.

\section{qRT-PCR analysis of drought-responsive genes and expression-QTL mapping at tillering stage}

Total RNA was isolated from well-watered leaves and drought stressed leaves using the TRI Reagent ${ }^{\circ}$ Solution (Ambion) and the Qiagen RNA Cleanup Protocol (RNeasy plant mini kit Qiagen). DNA contaminations were removed with the Ambion's DNA-free ${ }^{\text {Tw }}$ Kit. RNA quantity and integrity was confirmed loading the purified RNAs onto an Agilent ${ }^{\circ}$ RNA Nano 6000 chip and analyzed on an Agilent ${ }^{\circ} 2100$ BioAnalyzer according to the manufacturer's instructions. The cDNA was synthesized with Standard Reverse Transcription Protocol (Promega) and quantified by Qubit fluorometer ${ }^{\mathrm{m}}$ (Invitrogen).

The description of the selected genes and the complete set of primer pairs are listed in Additional file 8. The specificity and uniqueness of the primers and the amplicons were verified by amplicon sequencing. qRT-PCR reactions were performed with SYBR Green fluorescence detection in a qPCR thermal cycler (ABI PRISM 7300, Applied Biosystems). Each reaction was prepared using $3 \mu \mathrm{L}$ from a $2 \mathrm{ng} / \mu \mathrm{L}$ dilution of cDNA derived from the RT reaction, $12.5 \mu \mathrm{L}$ of SYBR Green PCR Master Mix (Qiagen), $1 \mu \mathrm{M}$ forward and reverse primers, in a total volume of $25 \mu \mathrm{L}$. The cycling conditions were: $10 \mathrm{~min}$ at $95^{\circ} \mathrm{C}$, followed by 40 cycles of $95^{\circ} \mathrm{C}$ for $15 \mathrm{sec}$ and $60^{\circ} \mathrm{C}$ for $1 \mathrm{~min}$ with the final dissociation at $95^{\circ} \mathrm{C}$ for $15 \mathrm{sec}$, $60^{\circ} \mathrm{C}$ for $30 \mathrm{sec}$ and $95^{\circ} \mathrm{C}$ for $15 \mathrm{sec}$. In order to identify a reference gene, the algorithm described by Aprile et al. [7] was used to find stably expressed transcripts using microarray data set. The best three ranking probe sets based on Coefficient of Variation (CV) and expression level were: Ta.12727.1.S1_at $(\mathrm{CV}=0.075$; Arabidopsis homolog = At2g42210, OEP16-3), Ta.9617.1.S1_at (CV = 0.090, At1g16700, NADH-ubiquinone oxidoreductase) and Ta.3583.1.A1_at (CV $=0.066$, At1g69120, AGL7). The CVs based on qRT-PCR data were: $\mathrm{CV}_{\text {OEP16-3 }}=0.018$, $\mathrm{CV}_{\text {NADH-ubiquinone oxidoreductase }}=0.020, \mathrm{CV}_{A G L 7}=0.033$. The CVs were lower than 0.050 and suitable as qRT-PCR reference genes. In the present work, we used OEP16-3 
as reference gene since it had the lowest $\mathrm{CV}$. Three biological replicates were used for quantification analysis. Melting curve analysis was performed to evaluate the presence of non-specific PCR products and primer dimers. Comparative $C_{t}$ method calculation steps to calculate the fold changes (FC) consist of the following three steps: 1) normalization to endogenous control by comparison of target gene and endogenous control $\Delta C_{t}=C_{t}$ target gene- $C_{t}$ endogenous gene; 2) normalization to calibrator sample $\left.\Delta \mathrm{C}_{\mathrm{t} \text { sample }}-\Delta \mathrm{Ct}_{\text {calibrator }}=\Delta \Delta \mathrm{C}_{\mathrm{t}} ; 3\right)$ using the formula $2^{-\Delta \Delta C t}$

To validate the microarray data, four probe sets were subjected to real-time qRT-PCR analysis: Ta.9335.1.S1_at (Acyl-CoA Oxidase, $A C X$ ), Ta.9184.1.S1_at (Multienzymatic complex, ECH/HCD), Ta.28367.1.S1_at (Acetyl-CoA acyltransferase, $A A T$ ), TaAffx.31738.1.S1_at (Acetate-CoA ligase/synthase, $A C L$ ).

The transcript HEL (High Expression Level) corresponding to the TaAffx.100436.1.S1_at probe set was selected as the most differentially expressed between Ofanto and Cappelli. The probe set sequence was used as a query in a blastn search against the Chinese Spring genomic DNA database of Cerealsdb database (http://www. cerealsdb.uk.net/CerealsDB/Documents/DOC_search_ reads.php) and the clone Contig444272 (1448 bp) was obtained (the sequence information are in Additional file 9). Two primers designed on Contig444272 (5'GTGGGTGGAGTGCATGTGGGTT-3' and 5'-ACCGG CGTTATCTGCGGTTGC-3') amplified a fragment of near $1000 \mathrm{bp}$ in Ofanto only, while no amplicons were found from genomic DNA of Cappelli. Amplicon identity was confirmed by sequencing. The PCR reactions were performed in $25 \mu \mathrm{L}$ volume with $80 \mathrm{ng}$ of template DNA, $1.5 \mathrm{mM} \mathrm{MgCl}_{2}, 0.2 \mathrm{mM}$ of dNTPs, $1 \mathrm{x}$ PCR-Buffer, $0.2 \mu \mathrm{M}$ of the two primers, and $1 \mathrm{U}$ of Platinum Taq DNA polymerase (Invitrogen); annealing temperature was set at $63^{\circ} \mathrm{C}$. This polymorphism was employed to map the corresponding locus in the frame of the previously published Ofanto x Cappelli genetic map [19,20], using the Kosambi mapping function within the JoinMap 4 software [78], considering a minimum LOD score $\left(\log _{10}\right.$ of the odds ratio) of 4.0 .

The expression of the gene corresponding to TaAffx. 100436.1.S1_at probe set was tested in response to drought on 80 Recombinant Inbreed Lines (RILs), representing the genetic variability of Ofanto $\mathrm{x}$ Cappelli segregating population. Two replications were considered for each RIL. QTL analysis was performed using the software package MapQTL ${ }^{\circ}$, version 5.0 [79] and the genetic map described by Marone et al. $[19,20]$. The logarithm of odds (LOD) profiles from simple interval mapping were inspected, and the marker closest to each LOD peak was selected as the cofactor to perform the multiple QTL mapping analysis. The LOD significance threshold levels of the respective traits were calculated with the permutation test option provided in MapQTL, using 10,000 permutations.

\section{Additional files}

Additional file 1: RNA degradation plot of the reference probe sets relative to GAPDH gene. On $x$-axis there are the 11 probes sorted by position along the GAPDH gene (from 5' to $3^{\prime}$ ). On $y$-axis is reported the relative expression value (scaled and shifted). Parallel lines mean that the RNA degradation is constant among samples.

Additional file 2: Validation of microarray data for four probe sets by qRT-PCR. In each graph the array data are plotted on the left and qRT-PCR data on the right. Blue bars represent Ofanto data. Red bars represent Cappelli data. CTRL expression data were used as baseline for fold change calculations. The log2 fold change is plotted on the $y$-axis. The Pearson correlation among microarray FC and GPCR FC is 0.753. ACS: acyl-CoA synthase; ACX: acyl-CoA oxidase; ECH: enoyl-CoA hydratase; HCD: L- $\beta$-hydroxyacyl-CoA dehydrogenase; AAT: acyl-CoA-acetyltransferase enzyme or thiolase.

Additional file 3: The list of the 9,012 differentially expressed genes in the experiment is sorted in column A. For each probe set the relative homologous Arabidopsis gene (column B), e-score (C), annotation (D), the mean expression level for each treatment (columns E-L) are reported. From column $\mathrm{M}$ to column $\mathrm{V}$ are reported the statistical comparison results. Empty cells mean not differentially expressed. Column W shows the QT-cluster number for each probe set.

Additional file 4: QT-clustering analysis obtained using the expression values of the 8,660 stress-related genes differentially expressed in at least one condition/genotype. The analyses was performed with a minimum cluster size of 30 and a correlation value of 0.80 . The four treatment conditions, grouped by genotypes, are plotted on $x$ axis. The relative expression level (for each probe set the data were normalized to the median expression level of the Ofanto Ctrl samples) is plotted on the $y$ axis. The lines represent the mean expression trend of all probe sets belonging to each cluster.

Additional file 5: The list of the 1,923 differentially expressed genes belonging to cluster $1,4,8,10,17,18,23,40$ and 47 sorted by QT-cluster number (column W). For each probe set the relative homologous Arabidopsis gene (column B), e-score (C), annotation (D), the mean expression level for each treatment (columns E-L) are reported.

From column $\mathrm{M}$ to column $\mathrm{V}$ are reported the statistical comparison results.

Additional file 6: Data from Affymetrix hybridization experiments expressed as Fold Change (FC) in black. In comparison with the FCs of microarray data, the FCs of qRT-PCR results from the experiment of young plants at tillering stage subjected to severe drought stress of $\mathbf{2 7 / 2 8} \%$ of RSWC are reported in blue. The results showed the differences in gene expression between Ofanto and Cappelli in non stressed/control (Ctrl) vs stressed/drought (Dr) condition in 3rd and 4th columns, while the FC values in the comparisons Ofanto stressed/drought (Dr) vs Cappelli stressed/drought (Dr) and Ofanto non stressed/control (Ctrl) vs Cappelli non stressed/control (Ctrl) are listed in the 5th and 6th columns.

Additional file 7: Alignment analysis of Contig444272 sequence (1,448 bp) with the homologous sequences i) Plant Transcript Assembly TA97230_4565 Sequence, Accession TA97230_4565 Triticum aestivum ii) TC419595 (from DFCI Wheat Gene Index, Release12.0) iii) probe set TaAffx.100436.1.S1_at of Wheat Affymetrix Chip. From the alignment, carried out using the AlignXprogramm (VectorNTI, Invitrogen), the results suggest that Contig444272 sequence contains an intron in the region from 372 to $493 \mathrm{bp}$.

Additional file 8: Primer lists used to find expression level polymorphisms and to validate microarray data.

Additional file 9: Nucleotide sequence (223 bp) of HEL gene obtained from CDNA of Ofanto and Cappelli by sequencing analysis in the first part of the table, and in the second part Contig444272 sequence (1448 bp) of Chinese spring obtained from cerealsDB database. 


\section{Competing interests}

The authors declare that no academic competing interests exist. The funding organizations had no role in study design or preparation of the manuscript.

\section{Authors' contributions}

AA interpreted the microarray results. AA and RP carried out the bioinformatic analyses on microarray data. $\mathrm{LH}, \mathrm{DM}$ and CM carried out the eQTL mapping. GMB and AMM assisted with material isolation from drought stressed plants and combined stressed plants. PR assisted with material isolation from heat stressed plants. RP carried out qRT-PCR and the searching analysis of internal reference genes. VC, AMM, CP, PR, LD, FR and LC participated in the design of the study. LC coordinated the study. LD linked expression data to fatty acid metabolism. AA, CM, CP and LC wrote the manuscript. All authors read and approved the final manuscript.

\section{Acknowledgements}

This work was supported by the Italian Ministry of Education, University and Research, project "AGROGEN", project "PON01_01145-ISCOCEM", PRIN 2010-2011 project "2010Z77XAX_002" and by the Italian Ministry of Agriculture, project "MAPPA 5A".

\section{Author details}

${ }^{1}$ Department of Biological and Environmental Sciences and Technologies, University of Salento, Prov.le Lecce Monteroni, I-73100 Lecce, Italy. ${ }^{2}$ Consiglio per la Ricerca e la Sperimentazione in Agricoltura, Genomics Research Centre, via San Protaso 302, I-29107 Fiorenzuola d'Arda (PC), Italy. ${ }^{3}$ Biotechnological Centre, Faculty of Agriculture, University of South Bohemia, CZ-370 05 České Budějovice, Czech Republic. ${ }^{4}$ Consiglio per la Ricerca e la Sperimentazione in Agricoltura, Cereal Research Centre, SS16 km 675, 71122 Foggia, Italy.

Received: 13 June 2013 Accepted: 18 November 2013 Published: 22 November 2013

\section{References}

1. Lobell DB, Schlenker W, Costa-Roberts J: Climate trends and global crop production since 1980. Science 2011, 333:616-620.

2. Lopes MS, Reynolds MP: Drought adaptive traits and wide adaptation in elite lines derived from resynthesized hexaploid wheat. Crop Sci 2011, 51:1617-1626

3. Rampino P: Drought stress response in wheat: physiological and molecular analysis of resistant and sensitive genotypes. Plant Cell Environ 2006, 29:2143-2152.

4. Lawlor DW: Genetic engineering to improve plant performance under drought: physiological evaluation of achievements, limitations, and possibilities. J Exp Bot 2013, 64:83-1008.

5. Umezawa T, Fujita M, Fujita Y, Yamaguchi-Shinozaki K, Shinozaki K: Engineering drought tolerance in plants: discovering and tailoring genes to unlock the future. Curr Opin Biotechnol 2006, 17:113-122.

6. Tuteja N, Sopory SK: Chemical signaling under abiotic stress environment in plants. Plant Signal Behav 2008, 3:525-536.

7. Aprile A, Mastrangelo AM, De Leonardis AM, Galiba G, Roncaglia E, Ferrari F, De Bellis L, Turchi L, Giuliano G, Cattivelli L: Transcriptional profiling in response to terminal drought stress reveals differential responses along the wheat genome. BMC Genomics 2009, 10:279-279.

8. Degenkolbe T, Do PT, Zuther E, Repsilber D, Walther D, Hincha DK, Kohl Kl: Expression profiling of rice cultivars differing in their tolerance to long-term drought stress. Plant Mol Biol 2009, 69:133-153.

9. Ergen NZ, Budak H: Sequencing over 13000 expressed sequence tags from six subtractive cDNA libraries of wild and modern wheats following slow drought stress. Plant Cell Environ 2009, 32:220-236.

10. Qin D, Wu H, Peng H, Yao Y, Ni Z, Li Z, Zhou C, Sun Q: Heat stress-responsive transcriptome analysis in heat susceptible and tolerant wheat (Triticum aestivum L.) by using Wheat Genome Array. BMC Genomics 2008, 9:432-432

11. Wahid A: Physiological implications of metabolite biosynthesis for net assimilation and heat-stress tolerance of sugarcane (Saccharum officinarum) sprouts. J Plant Res 2007, 120:219-228.

12. Atienza SG, Faccioli P, Perrotta G, Dalfino G, Zschiesche W, Humbeck K, Stanca AM, Cattivelli L: Large scale analysis of transcripts abundance in barley subjected to several single and combined abiotic stress conditions. Plant Sci 2004, 167:1359-1365.
13. Mittler R: Abiotic stress, the field environment and stress combination. Trends Plant Sci 2006, 11:15-19.

14. Rizhsky L, Liang HJ, Mittler R: The combined effect of drought stress and heat shock on gene expression in tobacco. Plant Physiol 2002, 130:1143-1151.

15. Rizhsky L, Liang HJ, Shuman J, Shulaev V, Davletova S, Mittler R: When Defense pathways collide. The response of Arabidopsis to a combination of drought and heat stress. Plant Physiol 2004, 134:1683-1696.

16. De Vita P, Nigro FP: Breeding progress in morpho-physiological, agronomical and qualitative traits of durum wheat cultivars released in Italy during the 20th century. Eur J Agron 2007, 26:39-53.

17. Rizza F, Ghashghaie J, Meyer S, Matteu L, Mastrangelo AM, Badeck F-W: Constitutive differences in water use efficiency between two durum wheat cultivars. Field Crop Res 2012, 125:49-60.

18. Panio R, Motzo R, Mastrangelo AM, Marone D, Cattivelli L, Giunta F, De Vita P: Molecular mapping of stomatal-conductance-related traits in durum wheat (Triticum turgidum ssp. durum). Ann Appl Biol 2013, 162:258-270.

19. Marone D, Laido G, Gadaleta A, Colasuonno P, Ficco DBM, Giancaspro A, Giove S, Panio G, Russo MA, De Vita P, Cattivelli L, Papa R, Blanco A, Mastrangelo AM: A high-density consensus map of $A$ and $B$ wheat genomes. Theor App/ Genet 2012, 125:1619-1638.

20. Marone D, Panio G, Ficco DBM, Russo MA, De Vita P, Papa R, Rubiales D, Cattivelli L, Mastrangelo AM: Characterization of wheat DArT markers: genetic and functional features. Mol Genet Genomics 2012, 287:741-753.

21. Wise RP, Caldo RA, Hong L, Shen L, Cannon E, Dickerson JA: BarleyBase/ PLEXdb. Methods Mol Biol 2007, 406:347-363.

22. Coram TE, Settles ML, Chen X: Transcriptome analysis of hightemperature adult-plant resistance conditioned by Yr39 during the wheat-Puccinia striiformis f. sp. tritici interaction. Mol Plant Pathol 2008, 9:479-493.

23. Benjamini $Y$, Hochberg Y: Controlling the False Discovery Rate - a Practical and Powerful Approach to Multiple Testing. J R Stat Soc Series B 1995, 57:289-300

24. Schroeder Jl, Allen GJ, Hugouvieux V, Kwak JM, Waner D: Guard cell signal transduction. Annu Rev Plant Physiol Plant Mol Biol 2001, 52:627-658.

25. Aubert Y: RD20, a stress-inducible caleosin, participates in stomatal control, transpiration and drought tolerance in Arabidopsis thaliana. Plant and Cell Physiology 2010, 51:1975-1987.

26. Partridge M, Murphy DJ: Roles of a membrane-bound caleosin and putative peroxygenase in biotic and abiotic stress responses in Arabidopsis. Plant Physiol Biochem 2009, 47:796-806.

27. Blee E: Impact of phyto-oxylipins in plant defense. Trends Plant Sci 2002, 7:315-321.

28. Feussner I, Wasternack C: The lipoxygenase pathway. Annu Rev Plant Biol 2002, 53:275-297.

29. Tuteja N, Sopory SK: Plant signaling in stress: G-protein coupled receptors, heterotrimeric G-proteins and signal coupling via phospholipases. Plant Signal Behav 2008, 3:79-86.

30. Park KY, Jung JY, Park J, Hwang JU, Kim YW, Hwang I, Lee Y: A role for phosphatidylinositol 3-phosphate in abscisic acid-induced reactive oxygen species generation in guard cells. Plant Physiol 2003, 132:92-98.

31. Chinnusamy V, Gong ZZ, Zhu JK: Abscisic acid-mediated epigenetic processes in plant development and stress responses. J Integr Plant Biol 2008, 50:1187-1195.

32. Alexandre C, Moller-Steinbach Y, Schonrock N, Gruissem W, Hennig L: Arabidopsis MSI1 Is Required for Negative Regulation of the Response to Drought Stress. Mol Plant 2009, 2:675-687.

33. Zhang Y, Feng SH, Chen FF, Chen HD, Wang J, McCall C, Xiong Y, Deng XW: Arabidopsis DDB1-CUL4 ASSOCIATED FACTOR1 forms a nuclear E3 ubiquitin ligase with DDB1 and CUL4 that is involved in multiple plant developmental processes. Plant Cell 2008, 20:1437-1455.

34. Xu N, Gao XQ, Zhao XY, Zhu DZ, Zhou LZ, Zhang XS: Arabidopsis AtVPS15 is essential for pollen development and germination through modulating phosphatidylinositol 3-phosphate formation. Plant Mol Biol 2011, 77:251-260.

35. Riera $M$, Redko $Y$, Leung J: Arabidopsis RNA-binding protein UBA2a relocalizes into nuclear speckles in response to abscisic acid. Febs Letters 2006, 580:4160-4165.

36. Li JX, Wang XQ, Watson MB, Assmann SM: Regulation of abscisic acidinduced stomatal closure and anion channels by guard cell AAPK kinase. Science 2000, 287:300-303. 
37. Li JX, Kinoshita T, Pandey S, Ng CKY, Gygi SP, Shimazaki K, Assmann SM: Modulation of an RNA-binding protein by abscisic-acid-activated protein kinase. Nature 2002, 418:793-797.

38. Li JX, Assmann SM: An abscisic acid-activated and calcium-independent protein kinase from guard cells of fava bean. Plant Cell 1996, 8:2359-2368.

39. Mustilli AC, Merlot S, Vavasseur A, Fenzi F, Giraudat J: Arabidopsis OST1 protein kinase mediates the regulation of stomatal aperture by abscisic acid and acts upstream of reactive oxygen species production. Plant Cell 2002, 14:3089-3099.

40. Yoshida R, Hobo T, Ichimura K, Mizoguchi T, Takahashi F, Aronso J, Ecker JR, Shinozaki K: ABA-activated SnRK2 protein kinase is required for dehydration stress signaling in Arabidopsis. Plant Cell Physiol 2002, 43:1473-1483.

41. Boudsocq M, Barbier-Brygoo H, Lauriere C: Identification of nine sucrose nonfermenting 1-related protein kinases 2 activated by hyperosmotic and saline stresses in Arabidopsis thaliana. J Biol Chem 2004, 279:41758-41766

42. Dong CH, Zolman BK, Bartel B, Lee B, Stevenson B, Agarwal M, Zhu JK. Disruption of Arabidopsis CHY1 Reveals an Important Role of Metabolic Status in Plant Cold Stress Signaling. Mol Plant 2009, 2:59-72.

43. Lucas KA, Filley JR, Erb JM, Graybill ER, Hawes JW: Peroxisomal metabolism of propionic acid and isobutyric acid in plants. J Biol Chem 2007, 282:24980-24989.

44. Mishra SK, Tripp J, Winkelhaus S, Tschiersch B, Theres K, Nover L, Scharf KD: In the complex family of heat stress transcription factors, HsfA1 has a unique role as master regulator of thermotolerance in tomato. Genes Dev 2002, 16:1555-1567.

45. Hayashi M: Plant peroxisomes: Molecular basis of the regulation of their functions. J Plant Res 2000, 113:103-109.

46. Allenbach $L$, Poirier $Y$ : Analysis of the alternative pathways for the betaoxidation of unsaturated fatty acids using transgenic plants synthesizing polyhydroxyalkanoates in peroxisomes. Plant Physiol 2000, 124:1159-1168.

47. Arent S, Christensen CE, Pye VE, Norgaard A, Henriksen A: The multifunctional protein in peroxisomal beta-oxidation: structure and substrate specificity of the Arabidopsis thaliana protein mfp2. J Biol Chem 2010, 285:24066-24077.

48. Eastmond PJ, Graham IA: Re-examining the role of the glyoxylate cycle in oilseeds. Trends Plant Sci 2001, 6:72-77.

49. De Bellis L, Picciarelli P, Pistelli L, Alpi A: Localization of glyoxylate-cycle marker enzymes in peroxisomes of senescent leaves and green cotyledons. Planta 1990, 180:435-439.

50. Pistelli L, Nieri B, Smith SM, Alpi A, DeBellis L: Glyoxylate cycle enzyme activities are induced in senescent pumpkin fruits. Plant Sci 1996, 119:23-29.

51. Balazadeh S, Riano-Pachon DM, Mueller-Roeber B: Transcription factors regulating leaf senescence in Arabidopsis thaliana. Plant Biology 2008, 10:63-75.

52. Breeze E, Harrison E, McHattie S, Hughes L, Hickman R, Hill C, Kiddle S, Kim YS, Penfold CA, Jenkins D, Zhang CJ, Morris K, Jenner C, Jackson S, Thomas B, Tabrett A, Legaie R, Moore JD, Wild DL, Ott S, Rand D, Beynon J, Denby K, Mead A, Buchanan-Wollaston V: High-resolution temporal profiling of transcripts during Arabidopsis leaf senescence reveals a distinct chronology of processes and regulation. Plant Cell 2011, 23:873-894.

53. Ulker B, Mukhtar MS, Somssich IE: The WRKY70 transcription factor of Arabidopsis influences both the plant senescence and defense signaling pathways. Planta 2007, 226:125-137.

54. Uauy C, Distelfeld A, Fahima T, Blechl A, Dubcovsky J: A NAC gene regulating senescence improves grain protein, zinc, and iron content in wheat. Science 2006, 314:1298-1301.

55. Jiang T, Zhang X-F, Wang X-F, Zhang D-P: Arabidopsis 3-Ketoacyl-CoA Thiolase-2 (KAT2), an enzyme of fatty acid b-oxidation, is involved in ABA signal transduction. Plant Cell Physiol 2011, 52:528-538.

56. Castillo MC, Leon J: Expression of the b-oxidation gene 3-ketoacyl-CoA thiolase 2 (KAT2) is required for the timely onset of natural and darkinduced leaf senescence in Arabidopsis. J Exp Bot 2008, 59:2171-2179.

57. $\mathrm{Xu} Z \mathrm{ZZ}$, Zhou GS: Combined effects of water stress and high temperature on photosynthesis, nitrogen metabolism and lipid peroxidation of a perennial grass Leymus chinensis. Planta 2006, 224:1080-1090.

58. Prasad PW, Staggenborg SA, Ristic Z: Impacts of drought and/or heat stress on physiological, developmental, growth, and yield processes of crop plants. In Response of crops to limited water: Understanding and modeling water stress effects on plant growth processes. Edited by Ahuja LR, Reddy VR, Saseendran SA, Yu Q, American Society of Agronomy. 2008:301-355.
59. Rampino P, Mita G, Fasano P, Borrelli GM, Aprile A, Dalessandro G, De Bellis L, Perrotta C: Novel durum wheat genes up-regulated in response to a combination of heat and drought stress. Plant Physiol Biochem 2012, $56: 72-78$

60. Senadheera P, Maathuis FJM: Differentially regulated kinases and phosphatases in roots may contribute to inter-cultivar difference in rice salinity tolerance. Plant Signal Behav 2009, 4:1163-1165.

61. Senadheera P, Maathuis FJM: Transcriptome analysis of membrane transporters in response to salinity stress. Methods Mol Biol 2012, 913:291-303.

62. Cubillos FA, Coustham V, Loudet O: Lessons from eQTL mapping studies: non-coding regions and their role behind natural phenotypic variation in plants. Curr Opin Plant Biol 2012, 15:192-198.

63. Ingvarsson PK, Street NR: Association genetics of complex traits in plants. New Phytologist 2011, 189:909-922.

64. Druka A, Potokina E, Luo Z, Jiang N, Chen X, Kearsey M, Waugh R: Expression quantitative trait loci analysis in plants. Plant Biotechnol $\mathrm{J}$ 2010, 8:10-27.

65. Vágújfalvi A, Galiba G, Cattivelli L, Dubcovsky J: The cold-regulated transcriptional activator $\mathrm{Cbf} 3$ is linked to the frost-tolerance locus Fr-A2 on wheat chromosome 5A. Mol Genet Genomics 2003, 269:60-67.

66. Francia E, Rizza F, Cattivelli L, Stanca AM, Galiba G, Tóth B, Hayes PM, Skinner JS, Pecchioni N: Two loci on chromosome $5 \mathrm{H}$ determine lowtemperature tolerance in a 'Nure' (winter) x 'Tremois' (spring) barley map. Theor Appl Genet 2004, 108:670-680

67. Shim J, Karim M: The control of mRNA stability in response to extracellular stimuli. Mol Cells 2002, 14:323-331.

68. Jordan MC, Somers DJ, Banks TW: Identifying regions of the wheat genome controlling seed development by mapping expression quantitative trait loci. Plant Biotechnol J 2007, 5:442-453.

69. Neumann K, Kobiljski B, Denčić SV, Varshney RK, BornerNeumann AB: Genome-wide association mapping: a case study in bread wheat (Triticum aestivum L.). Molecular Breeding 2011, 27:37-58.

70. Maccaferri M, Sanguineti MC, Demontis A, El-Ahmed A, Garcia del Moral L, Maalouf F, Nachit M, Nserallah N, Ouabbou H, Rhouma S, Royo C, Villegas D, Tuberosa R: Association mapping in durum wheat grown across a broad range of water regimes. J Exp Bot 2011, 62:409-438.

71. Zadoks JC: A decimal code for the growth stages of cereals. Weed Research 1997, 14:415-421.

72. Dash S, Van Hemert J, Hong L, Wise RP, Dickerson JA: PLEXdb: gene expression resources for plants and plant pathogens. Nucleic Acids Res 2012, 40:D1194-D1201.

73. Irizarry RA, Hobbs B, Collin F, Beazer-Barclay YD, Antonellis KJ, Scherf U, Speed TP: Exploration, normalization, and summaries of high density oligonucleotide array probe level data. Biostatistics 2003, 4:249-264.

74. Gautier L, Cope L, Bolstad BM, Irizarry RA: affy-analysis of Affymetrix GeneChip data at the probe level. Bioinformatics 2004, 20:307-315.

75. Yeung KY, Ruzzo WL: Principal component analysis for clustering gene expression data. Bioinformatics 2001, 17:763-774.

76. Heyer LJ, Kruglyak S, Yooseph S: Exploring expression data: Identification and analysis of coexpressed genes. Genome Res 1999, 9:1106-1115.

77. Ruepp A, Zollner A, Maier D, Albermann K, Hani J, Mokrejs M, Tetko I, Guldener U, Mannhaupt G, Munsterkotter M, Mewes HW: The FunCat, a functional annotation scheme for systematic classification of proteins from whole genomes. Nucleic Acids Res 2004, 32:5539-5545.

78. Van Ooijen JW: Joinmap 4: Software for the calculation of genetic linkage maps in experimental populations. Wageningen, Netherlands: Kyazma BV; 2006.

79. Van Ooijen JW, Voorips RE: JoinMap Version 3.0, Software for the calculation of genetic linkage maps. Wageningen, Netherlands: Kyazma BV; 2004.

doi:10.1186/1471-2164-14-821

Cite this article as: Aprile et al:: Different stress responsive strategies to drought and heat in two durum wheat cultivars with contrasting water use efficiency. BMC Genomics 2013 14:821. 